\title{
Analytical approximation of the neutrino oscillation matter effects at large $\theta_{13}$
}

\author{
Sanjib Kumar Agarwalla, ${ }^{a, 1}$ Yee $\mathrm{Kao}^{b}$ and Tatsu Takeuchi ${ }^{c, d}$ \\ ${ }^{a}$ Institute of Physics, Sachivalaya Marg, Sainik School Post, \\ Bhubaneswar 751005, Orissa, India \\ ${ }^{b}$ Department of Chemistry and Physics, Western Carolina University, \\ Cullowhee, NC 28723, U.S.A. \\ ${ }^{c}$ Center for Neutrino Physics, Physics Department, Virginia Tech, \\ Blacksburg, VA 24061, U.S.A. \\ ${ }^{d}$ Kavli Institute for the Physics and Mathematics of the Universe (WPI), The University of Tokyo, \\ Kashiwa-shi, Chiba-ken 277-8583, Japan \\ E-mail: sanjib@iopb.res.in, ykao@email.wcu.edu, takeuchi@vt.edu
}

ABSTRACT: We argue that the neutrino oscillation probabilities in matter are best understood by allowing the mixing angles and mass-squared differences in the standard parametrization to 'run' with the matter effect parameter $a=2 \sqrt{2} G_{F} N_{e} E$, where $N_{e}$ is the electron density in matter and $E$ is the neutrino energy. We present simple analytical approximations to these 'running' parameters. We show that for the moderately large value of $\theta_{13}$, as discovered by the reactor experiments, the running of the mixing angle $\theta_{23}$ and the CP violating phase $\delta$ can be neglected. It simplifies the analysis of the resulting expressions for the oscillation probabilities considerably. Approaches which attempt to directly provide approximate analytical expressions for the oscillation probabilities in matter suffer in accuracy due to their reliance on expansion in $\theta_{13}$, or in simplicity when higher order terms in $\theta_{13}$ are included. We demonstrate the accuracy of our method by comparing it to the exact numerical result, as well as the direct approximations of Cervera et al., Akhmedov et al., Asano and Minakata, and Freund. We also discuss the utility of our approach in figuring out the required baseline lengths and neutrino energies for the oscillation probabilities to exhibit certain desirable features.

Keywords: Neutrino Physics, Beyond Standard Model

ARXIV EPRINT: 1302.6773

\footnotetext{
${ }^{1}$ Corresponding author.
} 


\section{Contents}

1 Introduction 1

2 The approximation $\quad 2$

2.1 Diagonalization of the effective Hamiltonian 2

2.2 Effective running mixing angles 4

2.3 Neutrino case 4

2.4 Anti-neutrino case 5

2.5 The $\beta$-dependence of mixing parameters 6

$\begin{array}{lll}3 & \text { Demonstration of the accuracy of the approximation } & 7\end{array}$

4 Applications $\quad 11$

4.1 Determination of the mass hierarchy from $\nu_{e}$ oscillations 11

$\begin{array}{ll}4.2 \text { The "magic" baseline } & 15\end{array}$

$\begin{array}{lll}5 & \text { Summary } & 16\end{array}$

$\begin{array}{lr}\text { A Conventions, notation, and basic formulae } & 17\end{array}$

$\begin{array}{ll}\text { A.1 The PMNS matrix } & 17\end{array}$

$\begin{array}{lll}\text { A.2 Neutrino oscillation } & 18\end{array}$

A.3 Matter effects 20

B Jacobi method $\quad 21$

B.1 Setup 21

B.2 Diagonalization of a $2 \times 2$ matrix 22

B.3 Neutrino case 23

B.3.1 Mixing angles and mass-squared differences 23

$\begin{array}{lll}\text { B.3.2 First rotation } & 23\end{array}$

$\begin{array}{lll}\text { B.3.3 Second rotation } & 25\end{array}$

B.3.4 Absorption of $\phi^{\prime}$ into $\theta_{13} \quad 27$

$\begin{array}{lll}\text { B.4 Anti-neutrino case } & 29\end{array}$

$\begin{array}{llr}\text { B.4.1 First rotation } & 29\end{array}$

$\begin{array}{lll}\text { B.4.2 Second rotation } & 30\end{array}$

B.4.3 Absorption of $\bar{\phi}^{\prime}$ into $\theta_{13} \quad 32$

C Commutation of $R_{13}$ and $R_{23}$ through $R_{12}$

C.1 Neutrino case 34

C.2 Anti-neutrino case 36 


\section{Introduction}

When performing long-baseline neutrino oscillation experiments on the Earth with accelerator based beams, or when detecting atmospheric neutrinos coming from below, the neutrinos necessarily traverse the Earth's interior [1-8]. This makes the understanding of matter effects [9-12] on the neutrino oscillation probabilities an indispensable part of analyzing such experiments. These matter effects can of course be calculated numerically for arbitrary matter profiles, but approximate analytical expressions are useful not only for making initial estimates on the requirements one must place on long-baseline experiments, but in obtaining a deeper understanding of the physics involved.

The exact three-flavor neutrino oscillation probabilities in constant-density matter can be expressed analytically [12-23]. This requires the diagonalization of the $3 \times 3$ effective Hamiltonian in matter whose ee-element in the flavor basis is shifted by $a=2 \sqrt{2} G_{F} N_{e} E$, where $N_{e}$ is the electron density and $E$ is the neutrino energy. The eigenvalues of the effective Hamiltonian yield the effective neutrino mass-squared differences in matter, ${ }^{1}$ while the diagonalization matrix is multiplied with the vacuum neutrino mixing matrix to yield its in-matter counterpart. Many authors adopt the standard vacuum parameterization of the mixing matrix to the matter version, and absorb matter effects into shifts of the mixing angles and $\mathrm{CP}$ violating phase, yielding the effective values of these parameters in matter $[13,14,16,22]$. Thus, the neutrino oscillation probabilities in matter can be obtained from those in vacuum by simply replacing the mass-squared differences, mixing angles, and $\mathrm{CP}$ violating phase with their effective values. Unfortunately, the final exact expressions for the neutrino oscillation probabilities obtained this way are too complicated to yield physical insight, especially if re-expressed in terms of the vacuum parameters.

Consequently, various analytical approximations have been devised to better understand the physics potential of various neutrino experiments [12, 22, 25-32]. These approximations relied on expansions in the small parameters $\alpha=\delta m_{21}^{2} / \delta m_{31}^{2} \approx 0.03$ and/or $s_{13}=\sin \theta_{13}$ in one form or another, a systematic treatment of which can be found in ref. [29]. In some cases the matter-effect parameter $a=2 \sqrt{2} G_{F} N_{e} E$ was also assumed to be small $[12,26]$. For instance, the formula of Cervera et al. in ref. [27] and that of Ahkmedov et al. in ref. [29] include terms of order $O\left(\alpha^{2}\right), O\left(\alpha s_{13}\right)$, and $O\left(s_{13}^{2}\right)$. Unfortunately, the accuracies of these formulae suffer when the value of $\theta_{13}$ is as large as was measured by Daya Bay [33, 34] and RENO [35], consistent with both earlier and later determinations by T2K [36], MINOS [37, 38], and Double Chooz [39, 40]. Given that the current world average of $s_{13}=\sin \theta_{13}$ is about 0.15 [41], the terms included are not all of the same order. Asano and Minakata [32] have derived the order $O\left(\alpha s_{13}^{2}\right)$ and $O\left(s_{13}^{4}\right)$ corrections to the Cervera et al. formula, but the simplicity of the original expressions is lost. Further improvements in accuracy are possible at the expense of simplicity, as was shown by Freund in ref. [22] where an approximate expression for the oscillation probability $P\left(\nu_{e} \rightarrow \nu_{\mu}\right)$ including all orders of $\theta_{13}$ was derived.

\footnotetext{
${ }^{1}$ The cubic characteristic equation for the eigenvalues of the effective Hamiltonian can be solved analytically using Cardano's formula [24].
} 
In previous papers $[42,43]$, we had argued the advantage of not expressing the neutrino oscillation probabilities in matter directly in terms of the vacuum parameters, but to maintain their expressions in terms of the effective parameters in matter which 'ran' with the parameter $a=2 \sqrt{2} G_{F} N_{e} E$. Further, it was shown that the Jacobi method [44] could be utilized to find approximate expressions for the 'running' parameters in a systematic fashion, leading to fairly simple and compact expressions. In particular, it was shown that the effective values of $\theta_{23}$ and the $\mathrm{CP}$ violating phase $\delta$ do not 'run' to the order considered, maintaining their vacuum values at all neutrino energies and baselines. (The non-running of $\theta_{23}$ and $\delta$ has also been discussed in ref. [17].) The $a$-dependence of the resulting expressions for the oscillation probabilities in terms of the approximate running parameters could be analyzed in a simple manner, facilitating the understanding of matter effects.

The approximation of refs. [42, 43] worked extremely well except when $\theta_{13}$ was very small, a possibility that could not be ignored until the Daya Bay/RENO measurements. In this paper, we reintroduce the method with further refinements which improve the accuracy of the approximation for large $\theta_{13}$, while maintaining its ease of use.

This paper is organized as follows. In section 2, we explain our approach to the matter effect problem, and list all the formulae necessary to calculate the approximate 'running' parameters in our approach. Approximate oscillation probabilities are obtained by replacing the mass-squared differences and mixing angles in the vacuum oscillation probabilities with their effective 'running' values. In section 3 , we demonstrate the accuracy of our approximation at various baseline lengths, different mass hierarchies, and different values of the $\mathrm{CP}$ violating phase $\delta$. Comparisons with the approximations of Cervera et al. [27], Akhmedov et al. [29], Asano-Minakata [32], and Freund [22] are also made. In section 4, we show how simple calculations using our approximation can be used to derive the baselines and energies at which the oscillation probabilities exhibit desirable features. We conclude in section 5. Detailed derivation of our approximation is given in appendices B and $\mathrm{C}$.

\section{The approximation}

In the following, we use the conventions and notation reviewed in appendix A.

\subsection{Diagonalization of the effective Hamiltonian}

If the matter density along the baseline is constant, ${ }^{2}$ the effective Hamiltonian which governs the evolution of neutrino flavor in matter is given by

$$
H_{a}=U\left[\begin{array}{ccc}
0 & 0 & 0 \\
0 & \delta m_{21}^{2} & 0 \\
0 & 0 & \delta m_{31}^{2}
\end{array}\right] U^{\dagger}+\left[\begin{array}{lll}
a & 0 & 0 \\
0 & 0 & 0 \\
0 & 0 & 0
\end{array}\right],
$$

where $U$ is the neutrino mixing matrix in vacuum, and

$$
a=2 \sqrt{2} G_{F} N_{e} E=7.63 \times 10^{-5}\left(\mathrm{eV}^{2}\right)\left(\frac{\rho}{\mathrm{g} / \mathrm{cm}^{3}}\right)\left(\frac{E}{\mathrm{GeV}}\right) .
$$

\footnotetext{
${ }^{2}$ At baseline length $L=10690 \mathrm{~km}$ or longer, the neutrino beam crosses the core-mantle-boundary and experiences a sudden jump in matter density. See ref. [46] for treatments of non-adiabatic transitions.
} 


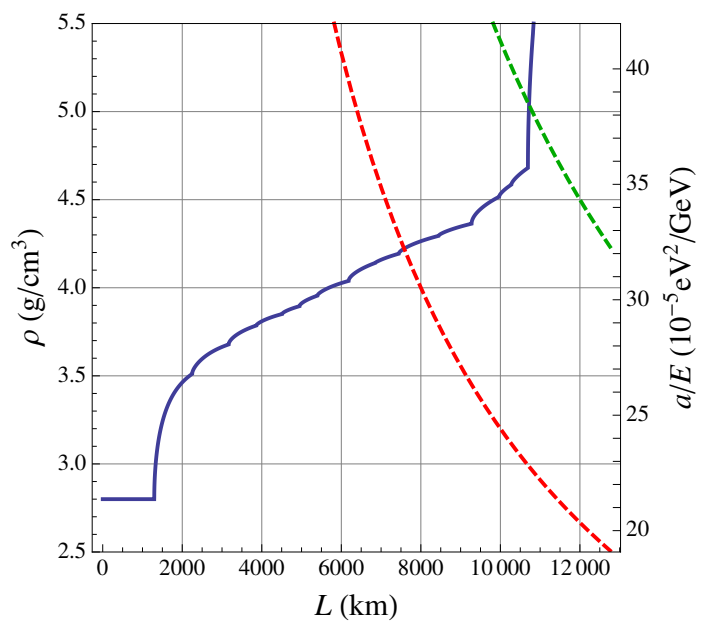

Figure 1. The dependence of the line-averaged mass density $\rho$ on the baseline length $L$ based on the Preliminary Reference Earth Model [45]. The labels on the right edge of the frame indicate the corresponding values of $a / E$. The green and red dashed lines indicate $\rho L=54000 \mathrm{~km} \cdot \mathrm{g} / \mathrm{cm}^{3}$ and $\rho L=32000 \mathrm{~km} \cdot \mathrm{g} / \mathrm{cm}^{3}$, respectively, which are conditions that will be discussed in section 4 .

Here, $N_{e}$ is the electron number density, $\rho$ the matter mass density along the baseline, and $E$ the neutrino energy. The above term appearing in the ee-component of $H_{a}$ is due to the interaction of $\nu_{e}$ with the electrons in matter via $W$-exchange, and eq. (2.2) assumes $N_{e}=N_{p}=N_{n}$ in Earth matter. It also assumes $E \ll M_{W}$ since the $W$-exchange interaction is approximated by a point-like four-fermion interaction. $Z$-exchange effects are flavor universal and only contribute a term proportional to the unit matrix to $H_{a}$, which can be dropped.

If we write the eigenvalues of $H_{a}$ as $\lambda_{i}(i=1,2,3)$ and the diagonalization matrix as $\tilde{U}$, that is

$$
H_{a}=\tilde{U}\left[\begin{array}{ccc}
\lambda_{1} & 0 & 0 \\
0 & \lambda_{2} & 0 \\
0 & 0 & \lambda_{3}
\end{array}\right] \tilde{U}^{\dagger}
$$

then the neutrino oscillation probabilities in matter are obtained by simply taking their expressions in vacuum and replacing the elements of the mixing matrix $U$ and the masssquare differences $\delta m_{i j}^{2}$ with their effective 'running' values in matter [9-11]:

$$
U_{\alpha i} \rightarrow \tilde{U}_{\alpha i}, \quad \delta m_{i j}^{2} \rightarrow \delta \lambda_{i j} \equiv \lambda_{i}-\lambda_{j}
$$

Note that $a$ is $E$-dependent, which means that both $\tilde{U}_{\alpha i}$ and $\delta \lambda_{i j}$ are also $E$-dependent. They also depend on the baseline length $L$ since the average matter density $\rho$ along a baseline varies with $L$. The $L$-dependence of the average $\rho$ and the corresponding value of $a / E$ are shown in figure 1 . 
For anti-neutrino beams, the flavor-evolution Hamiltonian in matter is

$$
\bar{H}_{a}=U^{*}\left[\begin{array}{ccc}
0 & 0 & 0 \\
0 & \delta m_{21}^{2} & 0 \\
0 & 0 & \delta m_{31}^{2}
\end{array}\right] U^{\mathrm{T}}+\left[\begin{array}{ccc}
-a & 0 & 0 \\
0 & 0 & 0 \\
0 & 0 & 0
\end{array}\right]
$$

In comparison to eq. (2.1), the $\mathrm{CP}$ violating phase $\delta$ in $U$ and the matter-effect term $a$ both acquire minus signs. Let us write the eigenvalues of $\bar{H}_{a}$ as $\bar{\lambda}_{i}(i=1,2,3)$ and the diagonalization matrix as $\tilde{U}$, that is

$$
\bar{H}_{a}=\breve{U}^{*}\left[\begin{array}{ccc}
\bar{\lambda}_{1} & 0 & 0 \\
0 & \bar{\lambda}_{2} & 0 \\
0 & 0 & \bar{\lambda}_{3}
\end{array}\right] \widetilde{U}^{\mathrm{T}}
$$

Note that the tilde above $\widetilde{U}$ here is flipped to distinguish it from $\widetilde{U}$ in eq. (2.3). The antineutrino oscillation probabilities in matter are then obtained by making the replacements

$$
U_{\alpha i} \rightarrow \widetilde{U}_{\alpha i}, \quad \delta m_{i j}^{2} \rightarrow \delta \bar{\lambda}_{i j} \equiv \bar{\lambda}_{i}-\bar{\lambda}_{j}
$$

in the vacuum expressions.

\subsection{Effective running mixing angles}

While it is possible to write down exact analytical expressions for $\tilde{U}_{\alpha i}$ and $\delta \lambda_{i j}$, as well as their anti-neutrino counterparts [16], simpler and more transparent approximate expressions are often desirable. One popular approach is to expand the probability formulae in terms of small parameters such as $\delta m_{21}^{2} /\left|\delta m_{31}^{2}\right|$ and $\theta_{13}$. Our approach, however, utilizes the Jacobi method [44]. Instead of obtaining approximations for the probabilities directly, we derived the approximations for the effective mixing parameters. In the following two sections, we list the expressions necessary to calculate the effective running mixing angles and the effective running mass-squared differences for the neutrino and anti-neutrino cases separately. Detailed derivation of our approximation is given in appendix B.

\subsection{Neutrino case}

We first recognize that the mixing matrix in matter can be parameterized in the same fashion as in the vacuum case:

$$
\widetilde{U}=R_{23}\left(\tilde{\theta}_{23}, 0\right) R_{13}\left(\tilde{\theta}_{13}, \tilde{\delta}\right) R_{12}\left(\tilde{\theta}_{12}, 0\right)
$$

The effective mixing angles can be approximated by

$$
\begin{aligned}
\tilde{\theta}_{12} & \approx \theta_{12}^{\prime}, \\
\tilde{\theta}_{13} & \approx \theta_{13}^{\prime}, \\
\tilde{\theta}_{23} & \approx \theta_{23}, \\
\widetilde{\delta} & \approx \delta,
\end{aligned}
$$


where $\theta_{12}^{\prime}$ and $\theta_{13}^{\prime}$ are given by

$$
\begin{aligned}
\tan 2 \theta_{12}^{\prime} & =\frac{\left(\delta m_{21}^{2} / c_{13}^{2}\right) \sin 2 \theta_{12}}{\left(\delta m_{21}^{2} / c_{13}^{2}\right) \cos 2 \theta_{12}-a} \\
\tan 2 \theta_{13}^{\prime} & =\frac{\left(\delta m_{31}^{2}-\delta m_{21}^{2} s_{12}^{2}\right) \sin 2 \theta_{13}}{\left(\delta m_{31}^{2}-\delta m_{21}^{2} s_{12}^{2}\right) \cos 2 \theta_{13}-a} .
\end{aligned}
$$

while the angle $\theta_{23}$ and the CP-violating phase $\delta$ at kept at their vacuum values [17].

The eigenvalues $\lambda_{i}(i=1,2,3)$ of $H_{a}$ are also given approximate running expressions:

$$
\begin{aligned}
& \lambda_{1} \approx \lambda_{-}^{\prime}, \\
& \lambda_{2} \approx \lambda_{\mp}^{\prime \prime}, \\
& \lambda_{3} \approx \lambda_{ \pm}^{\prime \prime},
\end{aligned}
$$

where the upper(lower) sign is for the normal(inverted) hierarchy, with

$$
\begin{aligned}
& \lambda_{ \pm}^{\prime} \equiv \frac{\left(\delta m_{21}^{2}+a c_{13}^{2}\right) \pm \sqrt{\left(\delta m_{21}^{2}-a c_{13}^{2}\right)^{2}+4 a c_{13}^{2} s_{12}^{2} \delta m_{21}^{2}}}{2}, \\
& \lambda_{ \pm}^{\prime \prime} \equiv \frac{\left[\lambda_{+}^{\prime}+\left(\delta m_{31}^{2}+a s_{13}^{2}\right)\right] \pm \sqrt{\left[\lambda_{+}^{\prime}-\left(\delta m_{31}^{2}+a s_{13}^{2}\right)\right]^{2}+4 a^{2} s_{12}^{\prime 2} c_{13}^{2} s_{13}^{2}}}{2},
\end{aligned}
$$

and $s_{12}^{\prime 2}=\sin ^{2} \theta_{12}^{\prime}$. For the inverted hierarchy case, $\delta m_{31}^{2}<0$, the above expressions simplify to

$$
\lambda_{2} \approx \lambda_{+}^{\prime \prime} \approx \lambda_{+}^{\prime}, \quad \lambda_{3} \approx \lambda_{-}^{\prime \prime} \approx \delta m_{31}^{2}<0 .
$$

Thus, to take matter effects into account when calculating neutrino oscillation probabilities, all that is necessary is to take their expressions in terms of the mixing angles and CP-phase in vacuum as is, and replace the two angles as well as the mass-squared differences with their effective running values in matter: $\theta_{12} \rightarrow \theta_{12}^{\prime}, \theta_{13} \rightarrow \theta_{13}^{\prime}, \delta m_{i j}^{2} \rightarrow \delta \lambda_{i j}=\lambda_{i}-\lambda_{j}$. This simplifies the calculation considerably, and allows for a transparent understanding of how matter-effects affect neutrino oscillation by looking at the $a$-dependence of the effective parameters.

\subsection{Anti-neutrino case}

Similarly, in the anti-neutrino case, the mixing matrix can be parameterized by:

$$
\tilde{U}=R_{23}\left(\tilde{\theta}_{23}, 0\right) R_{13}\left(\tilde{\theta}_{13}, \widetilde{\delta}\right) R_{12}\left(\widetilde{\theta}_{12}, 0\right)
$$

Note that the sign in front of the matter effect parameter $a$ is flipped relative to the neutrino case, so these effective mixing angles will be different. Our approximation is given by

$$
\begin{aligned}
\widetilde{\theta}_{12} & \approx \bar{\theta}_{12}^{\prime}, \\
\widetilde{\theta}_{13} & \approx \bar{\theta}_{13}^{\prime}, \\
\tilde{\theta}_{23} & \approx \theta_{23}, \\
\widetilde{\delta} & \approx \delta,
\end{aligned}
$$


where

$$
\begin{aligned}
\tan 2 \bar{\theta}_{12}^{\prime} & =\frac{\left(\delta m_{21}^{2} / c_{13}^{2}\right) \sin 2 \theta_{12}}{\left(\delta m_{21}^{2} / c_{13}^{2}\right) \cos 2 \theta_{12}+a} \\
\tan 2 \bar{\theta}_{13}^{\prime} & =\frac{\left(\delta m_{31}^{2}-\delta m_{21}^{2} s_{12}^{2}\right) \sin 2 \theta_{13}}{\left(\delta m_{31}^{2}-\delta m_{21}^{2} s_{12}^{2}\right) \cos 2 \theta_{13}+a} .
\end{aligned}
$$

Again, $\theta_{23}$ and $\delta$ are unaffected while $\theta_{12}$ and $\theta_{13}$ are replaced by their effective running values in matter.

The eigenvalues $\bar{\lambda}_{i}(i=1,2,3)$ of $\bar{H}_{a}$ are given approximate running expressions as in the neutrino case. The three eigenvalues of the effective Hamiltonian are approximated by

$$
\begin{aligned}
& \bar{\lambda}_{1} \approx \bar{\lambda}_{\mp}^{\prime \prime}, \\
& \bar{\lambda}_{2} \approx \bar{\lambda}_{+}^{\prime}, \\
& \bar{\lambda}_{3} \approx \bar{\lambda}_{ \pm}^{\prime \prime},
\end{aligned}
$$

where the upper(lower) sign is for the normal(inverted) hierarchy, with

$$
\begin{aligned}
& \bar{\lambda}_{ \pm}^{\prime} \equiv \frac{\left(\delta m_{21}^{2}-a c_{13}^{2}\right) \pm \sqrt{\left(\delta m_{21}^{2}+a c_{13}^{2}\right)^{2}-4 a c_{13}^{2} s_{12}^{2} \delta m_{21}^{2}}}{2} \\
& \bar{\lambda}_{ \pm}^{\prime \prime} \equiv \frac{\left[\bar{\lambda}_{-}^{\prime}+\left(\delta m_{31}^{2}-a s_{13}^{2}\right)\right] \pm \sqrt{\left[\bar{\lambda}_{-}^{\prime}-\left(\delta m_{31}^{2}-a s_{13}^{2}\right)\right]^{2}+4 a^{2} \bar{c}_{12}^{\prime 2} c_{13}^{2} s_{13}^{2}}}{2}
\end{aligned}
$$

and $\bar{c}_{12}^{\prime 2}=\cos ^{2} \bar{\theta}_{12}^{\prime}$. For the normal hierarchy case, $\delta m_{31}^{2}>0$, the above expressions simplify to

$$
\bar{\lambda}_{1} \approx \bar{\lambda}_{-}^{\prime \prime} \approx \bar{\lambda}_{-}, \quad \bar{\lambda}_{3} \approx \bar{\lambda}_{+}^{\prime \prime} \approx \delta m_{31}^{2} .
$$

Thus, the calculation of matter effects for anti-neutrino beams entails the replacements $\theta_{12} \rightarrow \bar{\theta}_{12}^{\prime}, \theta_{13} \rightarrow \bar{\theta}_{13}^{\prime}, \delta m_{i j}^{2} \rightarrow \delta \bar{\lambda}_{i j}=\bar{\lambda}_{i}-\bar{\lambda}_{j}$.

\subsection{The $\beta$-dependence of mixing parameters}

We show plots depicting how our various effective parameters run with the matter-effect parameter $a$. Due to the wide separation in scale between $\delta m_{21}^{2}$ and $\delta m_{31}^{2}$, we find it convenient to introduce the parameter $\beta$ via $^{3}$

$$
\frac{a}{\left|\delta m_{31}^{2}\right|}=\varepsilon^{-\beta}, \quad \varepsilon \equiv \sqrt{\frac{\delta m_{21}^{2}}{\left|\delta m_{31}^{2}\right|}} \approx 0.17,
$$

and plot our effective running parameters as functions of $\beta$ instead of $a$. Here $\beta=0$ corresponds to $a=\left|\delta m_{31}^{2}\right|, \beta=-2$ to $a=\delta m_{21}^{2}$, and so on. The dependence of the effective mixing angles on $\beta$ are shown in figure 2 and that of the sines of twice these angles in figure 3. The $\beta$-dependence of approximate eigenvalues of the effective Hamiltonian are shown in figure 4.

\footnotetext{
${ }^{3}$ We avoid the use of the symbols $\alpha$ or $A$ since they often respectively denote $\delta m_{21}^{2} / \delta m_{31}^{2}$ and $a / \delta m_{31}^{2}$ in the literature.
} 


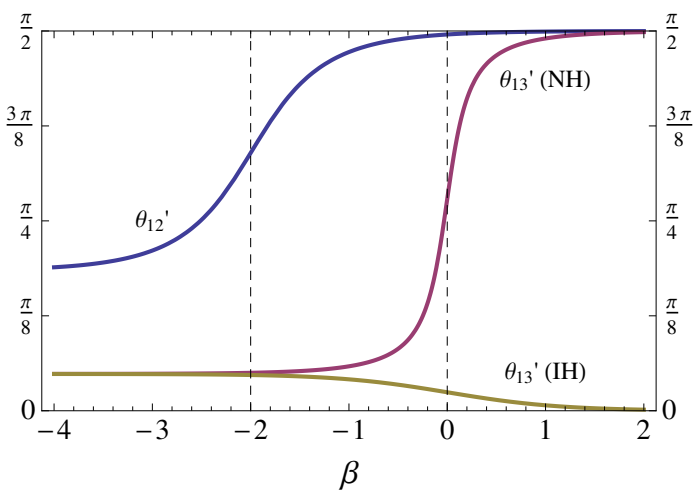

(a) neutrino mixing angles

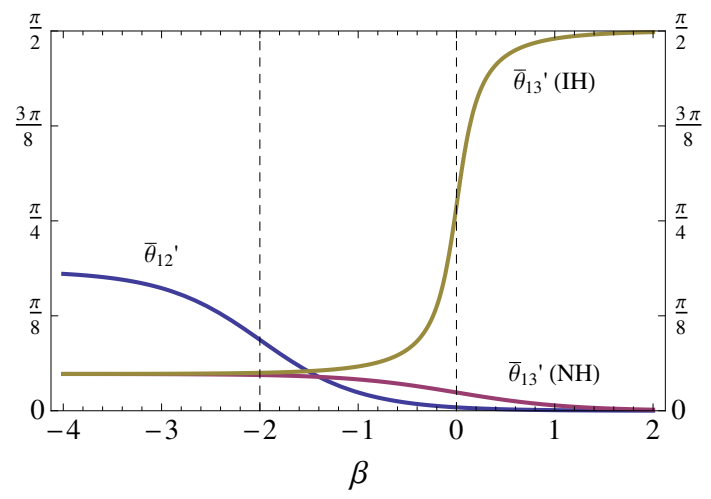

(b) anti-neutrino mixing angles

Figure 2. The dependences of the effective mixing angles on $\beta=-\log _{\varepsilon}\left(a /\left|\delta m_{31}^{2}\right|\right)$ for the neutrino (a) and antineutrino (b) cases. $\beta=0$ corresponds to $a=\left|\delta m_{31}^{2}\right|$, and $\beta=-2$ to $a=\delta m_{21}^{2}$. The $\beta$ dependences of $\theta_{13}^{\prime}$ and $\bar{\theta}_{13}^{\prime}$ depend on the mass hierarchy: when $\delta m_{31}^{2}>0$ (normal hierarchy, NH) $\theta_{13}^{\prime}$ increases toward $\pi / 2$ whereas $\bar{\theta}_{13}^{\prime}$ decreases toward zero, while in the $\delta m_{31}^{2}<0$ case (inverted hierarchy, $\mathrm{IH})$, it is the other way around.

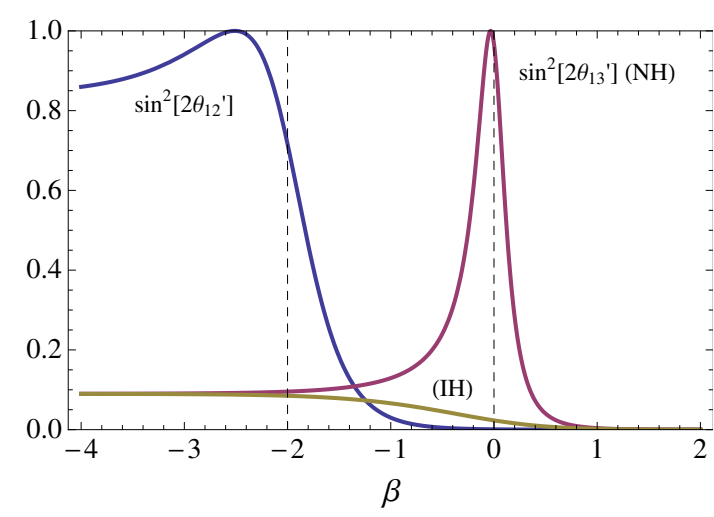

(a) neutrino case

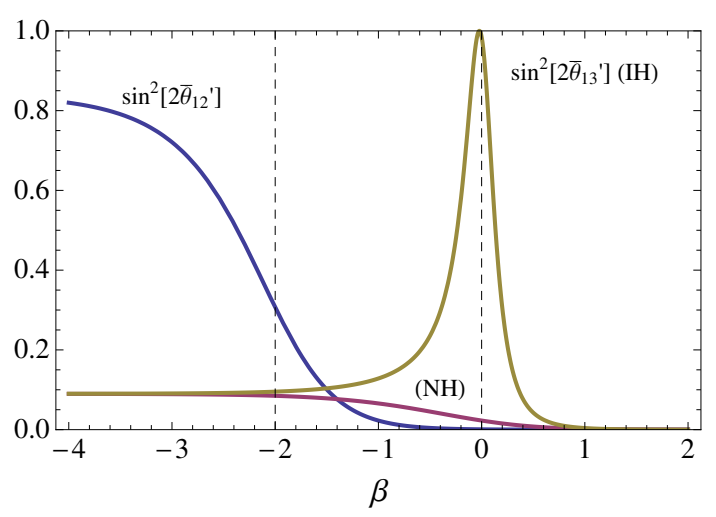

(b) anti-neutrino case

Figure 3. The $\beta$-dependences of the sines of twice the effective mixing angles for the neutrino (a) and antineutrino (b) cases. The difference in the behavior of the effective $\theta_{13}$ mixing angle for normal and inverted hierarchies will allow us to determine which is chosen by nature.

\section{Demonstration of the accuracy of the approximation}

In this section, we plot neutrino oscillation probabilities in several scenarios to demonstrate the accuracy of our approximation. As seen in the previous section, our formulae for both the neutrino and anti-neutrino cases are fairly compact and easy to code. In particular, the effective mixing angles for the neutrino and anti-neutrino cases can be calculated with the same code by simply flipping the sign of the matter-effect parameter $a$, cf. eqs. (2.10) and (2.16). The same can be said of $\lambda_{ \pm}^{\prime}$ and $\bar{\lambda}_{ \pm}^{\prime}$ defined in eqs. (2.12) and (2.18). In the case of $\lambda_{ \pm}^{\prime \prime}$ and $\bar{\lambda}_{ \pm}^{\prime \prime}$, one also needs to make the swap $\lambda_{+}^{\prime} \leftrightarrow \bar{\lambda}_{-}$but otherwise the code will be essentially the same. For the vacuum values of the mixing angles and mass-squared 


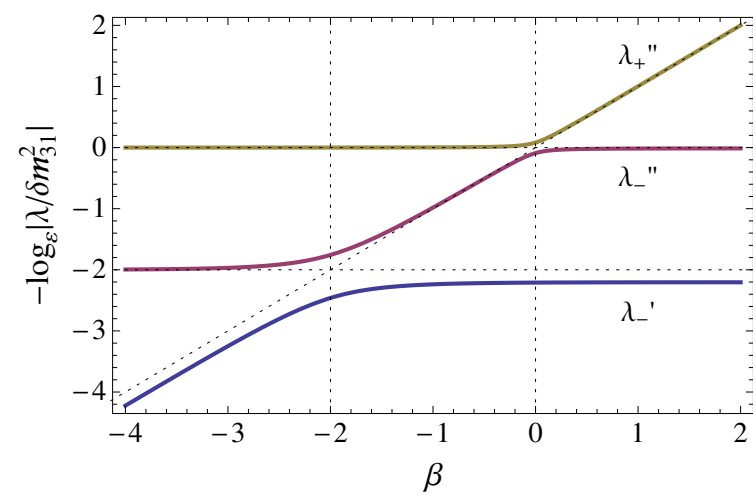

(a) neutrino, normal hierarchy

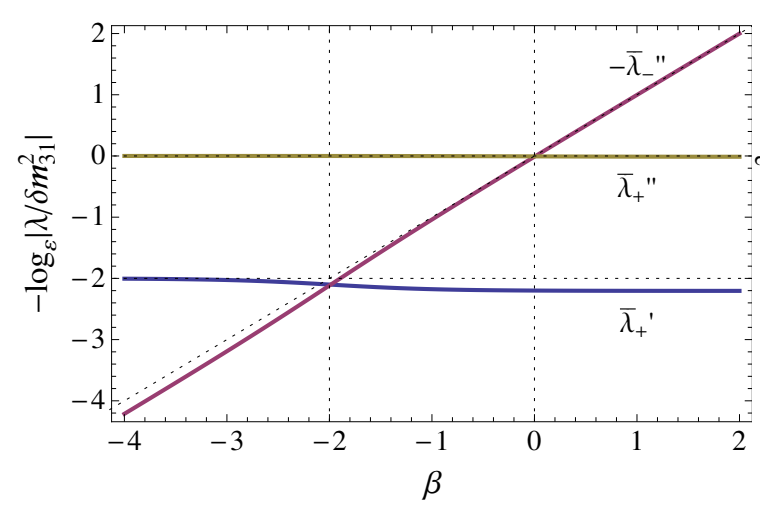

(c) anti-neutrino, normal hierarchy

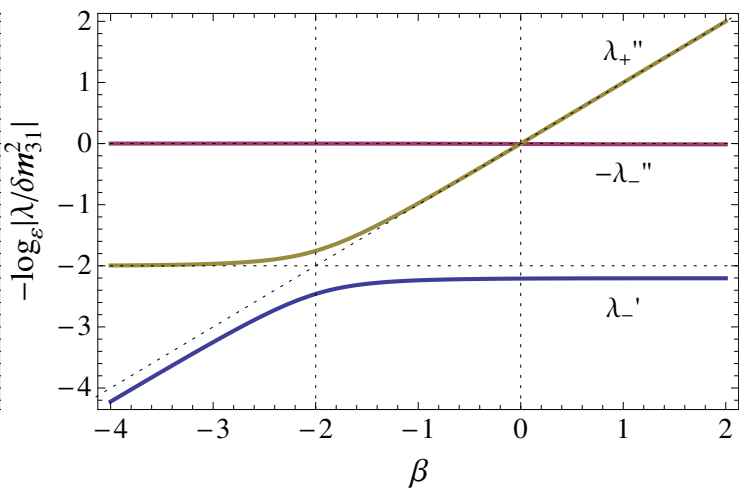

(b) neutrino, inverted hierarchy

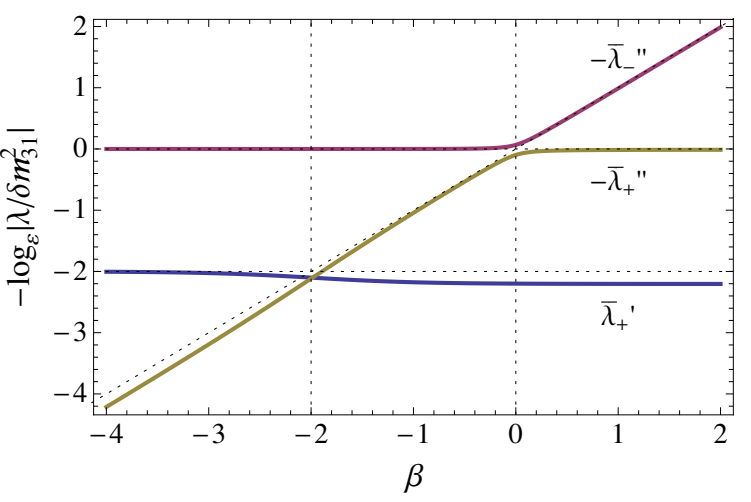

(d) anti-neutrino, inverted hierarchy

Figure 4. Dependence of the approximate eigenvalues of the effective Hamiltonian on $\beta=$ $-\log _{\varepsilon}\left(a /\left|\delta m_{31}^{2}\right|\right)$ for the (a) neutrino normal hierarchy, (b) neutrino inverted hierarchy, (c) antineutrino normal hierarchy, and (d) anti-neutrino inverted hierarchy cases.

\begin{tabular}{|c|c|}
\hline$\delta m_{21}^{2}$ & $7.5 \times 10^{-5} \mathrm{eV}^{2}$ \\
\hline$\delta m_{31}^{2}$ & $2.47 \times 10^{-3} \mathrm{eV}^{2}$ \\
\hline $\sin ^{2} \theta_{23}$ & 0.5 \\
\hline $\sin ^{2} \theta_{12}$ & 0.3 \\
\hline $\sin ^{2} \theta_{13}$ & 0.023 \\
\hline
\end{tabular}

Table 1. Best-fit values of oscillation parameters taken from ref. [41].

differences, we use the global fit values from ref. [41] listed in table 1. All plots are generated assuming constant Earth matter density.

We begin by comparing our approximation to eq. (16) of Cervera et al. [27], eq. (3.5) of Akhmedov et al. [29], sum of eqs. (4.2) to (4.4) of Asano and Minakata [32], and eq. (36) of Freund [22]. Note that both Cervera et al. and Akhmedov et al. expand the oscillation probabilities to the same order, so their expressions are quite similar except for a minor difference: eq. (16) of Cervera et al. is the same as eq. (38) of Freund, while eq. (3.5) of Akhmedov et al. is obtained from the same by setting $\cos \theta_{13}=1$ while keeping $\sin \theta_{13}$ non-zero. 


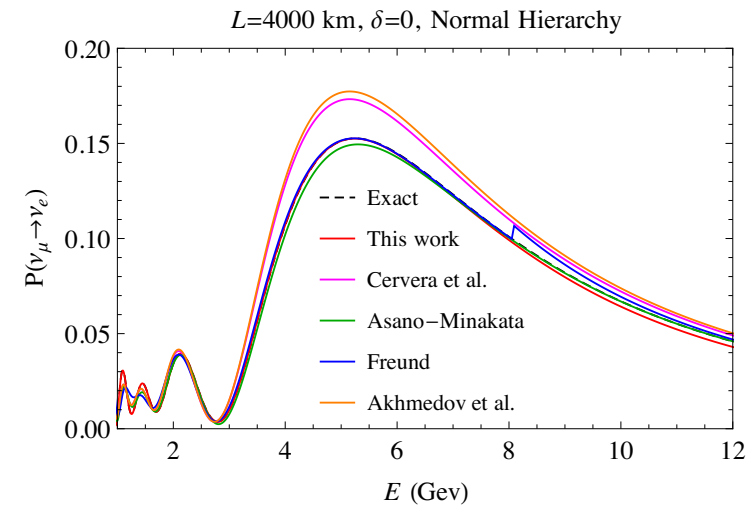

(a)

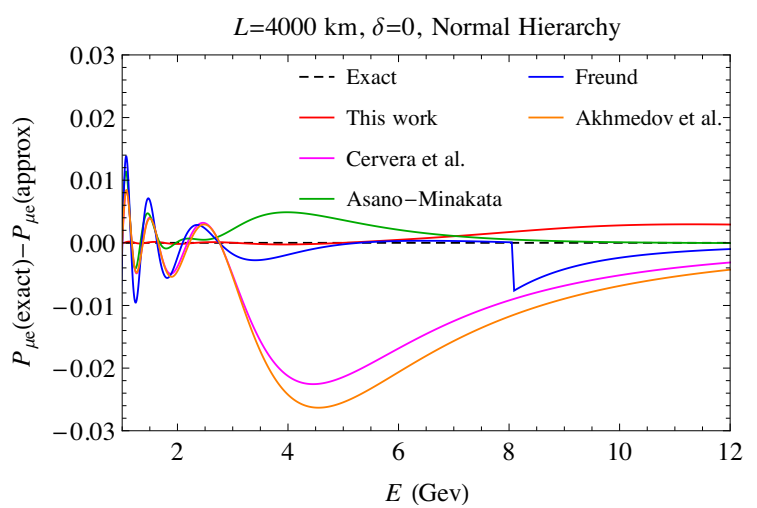

(b)

Figure 5. Comparison of the approximation formulae of Cervera et al., Akhmedov et al., AsanoMinakata, Freund, and this work at $L=4000 \mathrm{~km}$. In left panel, the dashed line gives the exact numerical result assuming the line-averaged constant matter density of $\rho=3.81 \mathrm{~g} / \mathrm{cm}^{3}$. This has been estimated using the PREM profile of the Earth [45].

In figure 5(a), we plot the approximate $\nu_{\mu} \rightarrow \nu_{e}$ oscillation probabilities calculated using these three approximations against the exact numerical result for the baseline length $L=4000 \mathrm{~km}$. This is the distance used by Asano and Minakata in ref. [32] to demonstrate the strength of their formula. The line-averaged constant Earth matter density ${ }^{4}$ for this baseline is $3.81 \mathrm{~g} / \mathrm{cm}^{3}$ which has been estimated using the Preliminary Reference Earth Model (PREM) [45]. We consider the normal hierarchy case, $\delta m_{31}^{2}>0$, with the CP violating phase $\delta$ set to zero. The differences between the exact and approximate formulae are plotted in figure 5(b). As can be seen, at this baseline, both the Asano-Minakata formula and our approximation work much better than the Cervera et al. or the Akhmedov et al. formulae. The Freund formula works well in the energy range $E \lesssim 8 \mathrm{GeV}$, but leads to a kink at $E \sim 8 \mathrm{GeV}$ due to some terms in the expression changing sign at $a=\left|\delta m_{13}^{2}\right| \cos 2 \theta_{13}$.

The comparison at a shorter baseline length of $L=810 \mathrm{~km}$, which is the distance from Fermilab to $\mathrm{NO} \nu \mathrm{A}$, is shown in figure 6 . There, all five approximations work well, with our approximation being the most accurate.

The situation changes at the longer baseline length of $L=8770 \mathrm{~km}$, which is the distance from CERN to Kamioka [47], as can be seen in figure 7. There, the Cervera et al. and the Akhmedov et al. formulae greatly overestimate $P\left(\nu_{\mu} \rightarrow \nu_{e}\right)$, while the AsanoMinakata formula leads to negative probability for $E \sim 4 \mathrm{GeV}$. The Freund formula is accurate up until $E \sim 7 \mathrm{GeV}$ where a kink occurs at $a=\left|\delta m_{13}^{2}\right| \cos 2 \theta_{13}$. In comparison, our approximation remains accurate for all energies.

The accuracy of our approximation for both the neutrino and anti-neutrino cases, and both mass hierarchies, for different values of the $\mathrm{CP}$ violating phase $\delta$, is demonstrated in figures 8 and 9 for the two baselines $L=1300 \mathrm{~km}$ and $L=2300 \mathrm{~km}$, respectively. These

\footnotetext{
${ }^{4}$ All the results presented in this paper have been derived assuming the line-averaged constant Earth matter density (based on the PREM profile) for a given baseline.
} 


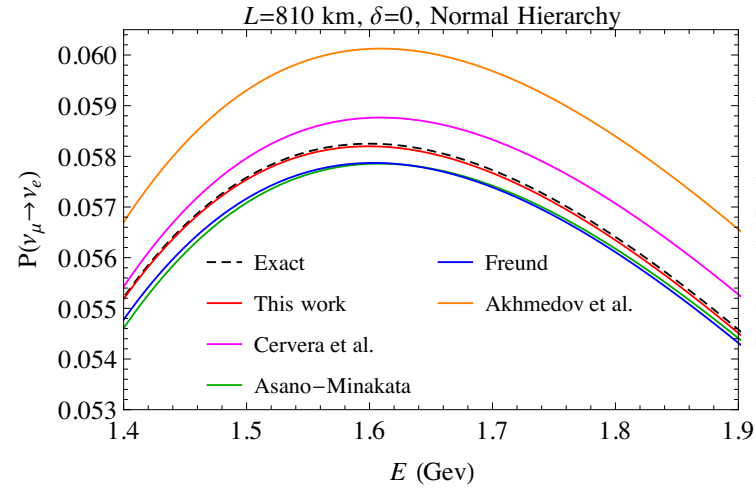

(a)

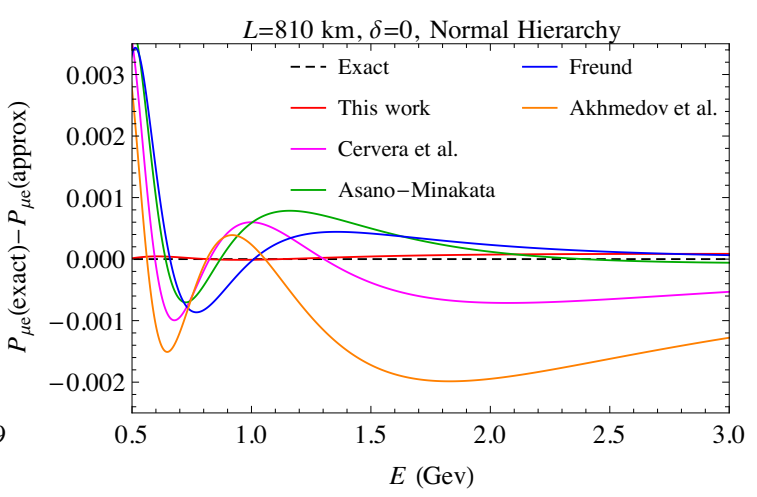

(b)

Figure 6. Comparison of the approximation formulae of Cervera et al., Akhmedov et al., AsanoMinakata, Freund, and this work at $L=810 \mathrm{~km}$, which is the distance from Fermilab to $\mathrm{NO} \nu \mathrm{A}$. In left panel, the dashed line gives the exact numerical result assuming the line-averaged constant matter density of $\rho=2.80 \mathrm{~g} / \mathrm{cm}^{3}$. This has been estimated using the PREM profile of the Earth [45].

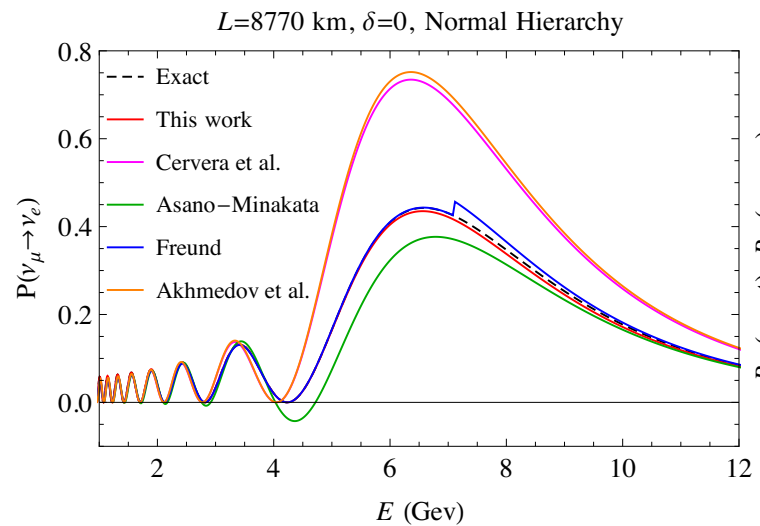

(a)

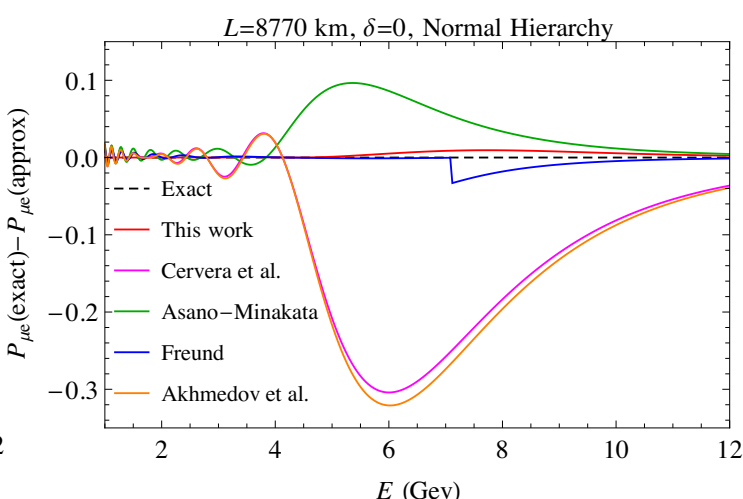

(b)

Figure 7. Comparison of the approximation formulae of Cervera et al., Akhmedov et al., AsanoMinakata, Freund, and this work at $L=8770 \mathrm{~km}$, which is the distance from CERN to Kamioka. In left panel, the dashed line gives the exact numerical result assuming the line-averaged constant matter density of $\rho=4.33 \mathrm{~g} / \mathrm{cm}^{3}$. This has been estimated using the PREM profile of the Earth [45]. Note that the Asano-Minakata formula gives negative probability for $E \sim 4 \mathrm{GeV}$.

distances correspond to those between Fermilab and Homestake $(1300 \mathrm{~km})$, and CERN and Pyhäsalmi $(2300 \mathrm{~km})$ [48]. As is evident, our approximation maintains its accuracy for all energy ranges and mass densities. 


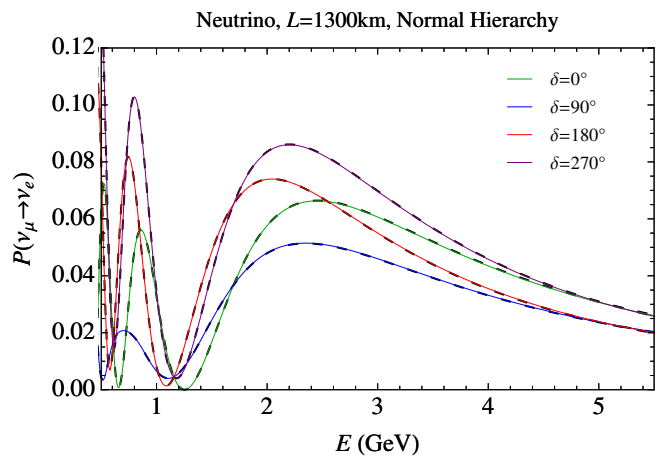

(a)

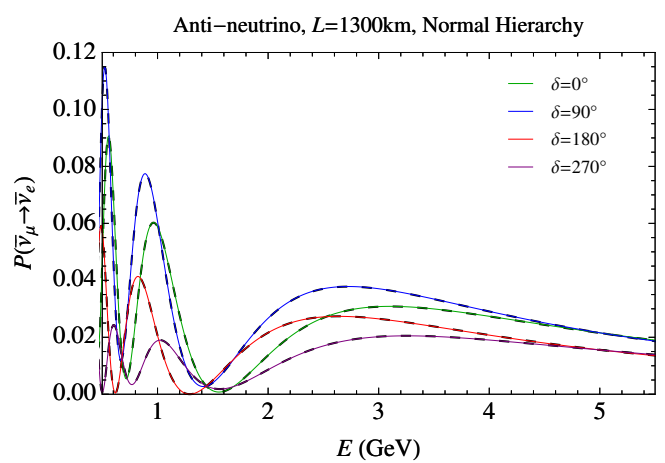

(c)

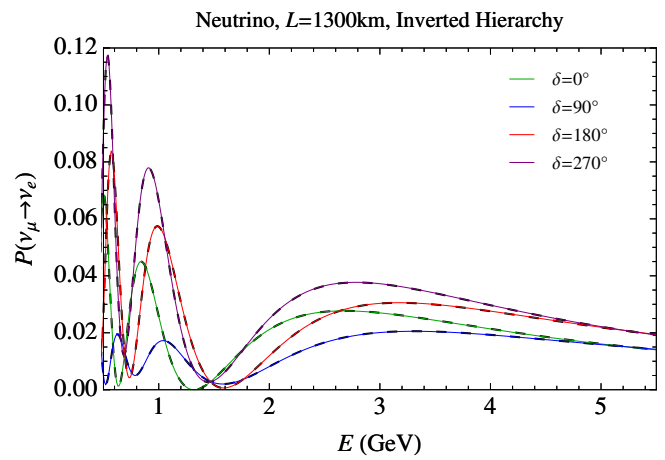

(b)

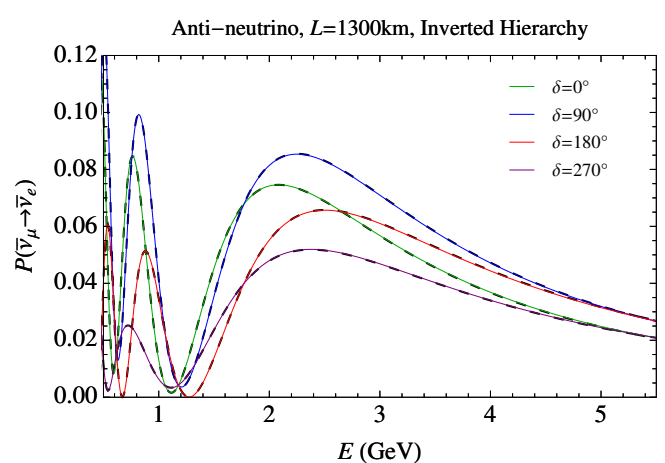

(d)

Figure 8. Comparison of our approximation formulae (colored) to the exact numerical results (black, dashed) for various values of the $\mathrm{CP}$ violating phase $\delta$ at $L=1300 \mathrm{~km}$. The line-averaged constant matter density for this baseline length is $\rho=2.87 \mathrm{~g} / \mathrm{cm}^{3}$.

\section{Applications}

\subsection{Determination of the mass hierarchy from $\nu_{e}$ oscillations}

Consider the $\nu_{e}$ survival probability in matter which is given by

$$
\begin{aligned}
& P\left(\nu_{e} \rightarrow \nu_{e}\right) \\
& =1-4\left|\tilde{U}_{e 2}\right|^{2}\left(1-\left|\tilde{U}_{e 2}\right|^{2}\right) \sin ^{2} \frac{\widetilde{\Delta}_{21}}{2}-4\left|\tilde{U}_{e 3}\right|^{2}\left(1-\left|\tilde{U}_{e 3}\right|^{2}\right) \sin ^{2} \frac{\widetilde{\Delta}_{31}}{2} \\
& +2\left|\tilde{U}_{e 2}\right|^{2}\left|\tilde{U}_{e 3}\right|^{2}\left(4 \sin ^{2} \frac{\widetilde{\Delta}_{21}}{2} \sin ^{2} \frac{\widetilde{\Delta}_{31}}{2}+\sin \tilde{\Delta}_{21} \sin \tilde{\Delta}_{31}\right) \\
& =1-4 c_{13}^{\prime 2} s_{12}^{\prime 2}\left(1-c_{13}^{\prime 2} s_{12}^{\prime 2}\right) \sin ^{2} \frac{\widetilde{\Delta}_{21}}{2}-\sin ^{2}\left(2 \theta_{13}^{\prime}\right) \sin ^{2} \frac{\widetilde{\Delta}_{31}}{2} \\
& +s_{12}^{\prime 2} \sin ^{2}\left(2 \theta_{13}^{\prime}\right)\left(2 \sin ^{2} \frac{\widetilde{\Delta}_{21}}{2} \sin ^{2} \frac{\widetilde{\Delta}_{31}}{2}+\frac{1}{2} \sin \widetilde{\Delta}_{21} \sin \widetilde{\Delta}_{31}\right) \\
& \stackrel{s_{12}^{\prime} \approx 1}{\longrightarrow} 1-\sin ^{2}\left(2 \theta_{13}^{\prime}\right)\left(\sin ^{2} \frac{\widetilde{\Delta}_{21}}{2}+\sin ^{2} \frac{\widetilde{\Delta}_{31}}{2}-2 \sin ^{2} \frac{\widetilde{\Delta}_{21}}{2} \sin ^{2} \frac{\widetilde{\Delta}_{31}}{2}-\frac{1}{2} \sin \widetilde{\Delta}_{21} \sin \widetilde{\Delta}_{31}\right) \\
& =1-\sin ^{2}\left(2 \theta_{13}^{\prime}\right) \sin ^{2} \frac{\widetilde{\Delta}_{32}}{2},
\end{aligned}
$$




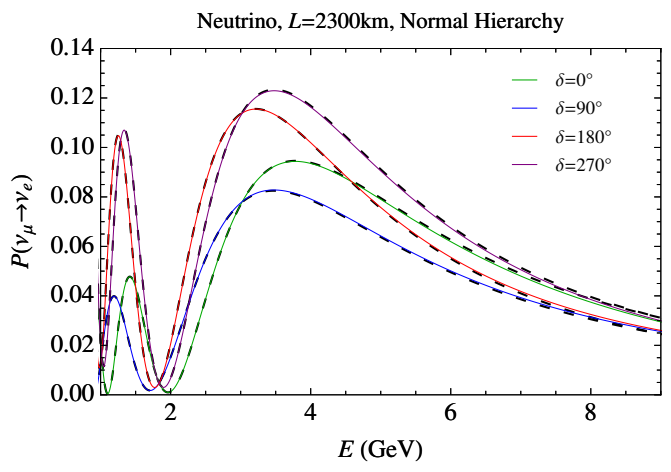

(a)

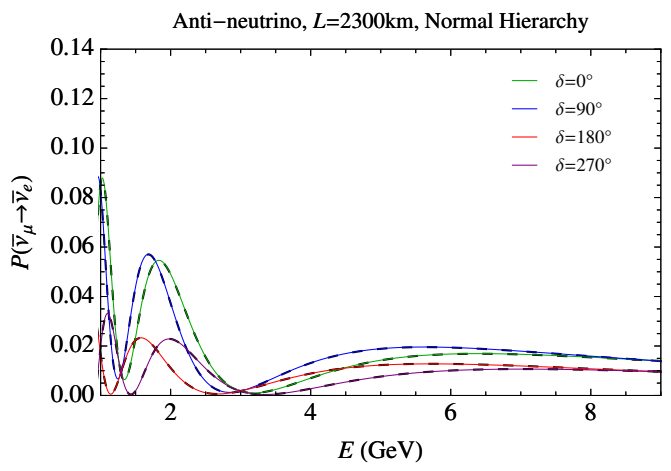

(c)

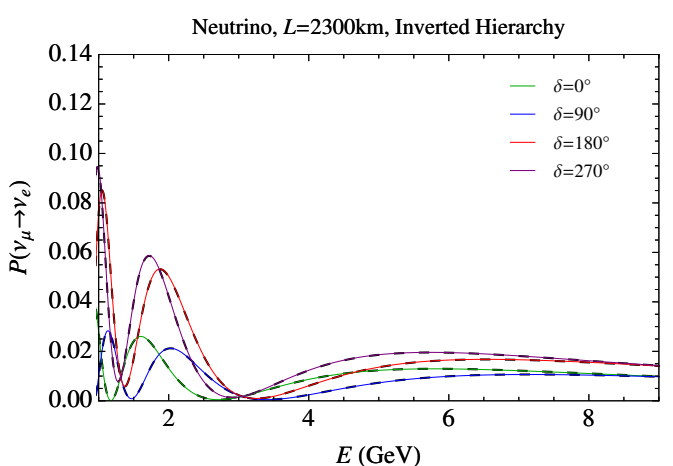

(b)

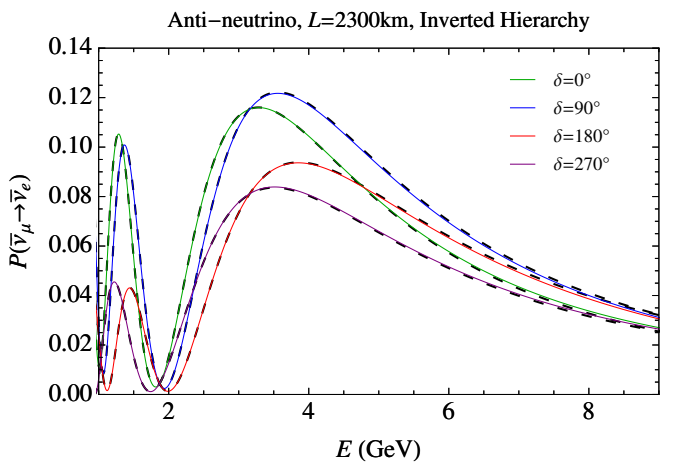

(d)

Figure 9. Comparison of our approximation formulae (colored) to the exact numerical results (black, dashed) for various values of the $\mathrm{CP}$ violating phase $\delta$ at $L=2300 \mathrm{~km}$. The line-averaged constant matter density for $L=2300 \mathrm{~km}$ is $\rho=3.54 \mathrm{~g} / \mathrm{cm}^{3}$.

where we have assumed that $a \gg \delta m_{21}^{2}$ so that $s_{12}^{\prime} \approx 1$ is a good approximation. Similarly, we find:

$$
\begin{aligned}
& P\left(\nu_{e} \rightarrow \nu_{\mu}\right) \\
& =4\left|\widetilde{U}_{e 2}\right|^{2}\left|\widetilde{U}_{\mu 2}\right|^{2} \sin ^{2} \frac{\widetilde{\Delta}_{21}}{2}+4\left|\tilde{U}_{e 3}\right|^{2}\left|\widetilde{U}_{\mu 3}\right|^{2} \sin ^{2} \frac{\widetilde{\Delta}_{31}}{2} \\
& +2 \Re\left(\widetilde{U}_{e 3}^{*} \widetilde{U}_{\mu 3} \widetilde{U}_{e 2} \widetilde{U}_{\mu 2}^{*}\right)\left(4 \sin ^{2} \frac{\widetilde{\Delta}_{21}}{2} \sin ^{2} \frac{\widetilde{\Delta}_{31}}{2}+\sin \widetilde{\Delta}_{21} \sin \widetilde{\Delta}_{31}\right) \\
& +4 \widetilde{J}_{(e, \mu)}\left(\sin ^{2} \frac{\widetilde{\Delta}_{21}}{2} \sin \widetilde{\Delta}_{31}-\sin ^{2} \frac{\widetilde{\Delta}_{31}}{2} \sin \widetilde{\Delta}_{21}\right) \\
& =4 s_{12}^{\prime 2} c_{13}^{\prime 2}\left(c_{12}^{\prime 2} c_{23}^{2}+s_{12}^{\prime 2} s_{13}^{\prime 2} s_{23}^{2}-2 s_{12}^{\prime} c_{12}^{\prime} s_{13}^{\prime} c_{23} s_{23} \cos \delta\right) \sin ^{2} \frac{\widetilde{\Delta}_{21}}{2}+4 s_{13}^{\prime 2} c_{13}^{\prime 2} s_{23}^{2} \sin ^{2} \frac{\widetilde{\Delta}_{31}}{2} \\
& +2 s_{12}^{\prime} s_{13}^{\prime} c_{13}^{\prime 2} s_{23}\left(c_{12}^{\prime} c_{23} \cos \delta-s_{12}^{\prime} s_{13}^{\prime} s_{23}\right)\left(4 \sin ^{2} \frac{\widetilde{\Delta}_{21}}{2} \sin ^{2} \frac{\widetilde{\widetilde{\Delta}}_{31}}{2}+\sin \widetilde{\Delta}_{21} \sin \widetilde{\Delta}_{31}\right)
\end{aligned}
$$




$$
\begin{aligned}
& -4 s_{12}^{\prime} c_{12}^{\prime} s_{13}^{\prime} c_{13}^{\prime 2} s_{23} c_{23} \sin \delta\left(\sin ^{2} \frac{\widetilde{\Delta}_{21}}{2} \sin \widetilde{\Delta}_{31}-\sin ^{2} \frac{\widetilde{\Delta}_{31}}{2} \sin \widetilde{\Delta}_{21}\right) \\
& \stackrel{s_{12}^{\prime} \approx 1}{\longrightarrow} s_{23}^{2} \sin ^{2}\left(2 \theta_{13}^{\prime}\right) \sin ^{2} \frac{\widetilde{\Delta}_{32}}{2} \\
& P\left(\nu_{e} \rightarrow \nu_{\tau}\right) \\
& =4\left|\tilde{U}_{e 2}\right|^{2}\left|\tilde{U}_{\tau 2}\right|^{2} \sin ^{2} \frac{\widetilde{\Delta}_{21}}{2}+4\left|\tilde{U}_{e 3}\right|^{2}\left|\tilde{U}_{\tau 3}\right|^{2} \sin ^{2} \frac{\widetilde{\Delta}_{31}}{2} \\
& +2 \Re\left(\widetilde{U}_{e 3}^{*} \tilde{U}_{\tau 3} \widetilde{U}_{e 2} \widetilde{U}_{\tau 2}^{*}\right)\left(4 \sin ^{2} \frac{\widetilde{\Delta}_{21}}{2} \sin ^{2} \frac{\widetilde{\Delta}_{31}}{2}+\sin \tilde{\Delta}_{21} \sin \widetilde{\Delta}_{31}\right) \\
& +4 \widetilde{J}_{(e, \tau)}\left(\sin ^{2} \frac{\widetilde{\Delta}_{21}}{2} \sin \tilde{\Delta}_{31}-\sin ^{2} \frac{\widetilde{\Delta}_{31}}{2} \sin \widetilde{\Delta}_{21}\right) \\
& =4 s_{12}^{\prime 2} c_{13}^{\prime 2}\left(c_{12}^{\prime 2} s_{23}^{2}+s_{12}^{\prime 2} s_{13}^{\prime 2} c_{23}^{2}-2 s_{12}^{\prime} c_{12}^{\prime} s_{13}^{\prime} s_{23} c_{23} \cos \delta\right) \sin ^{2} \frac{\widetilde{\Delta}_{21}}{2}+4 s_{13}^{\prime 2} c_{13}^{\prime 2} c_{23}^{2} \sin ^{2} \frac{\widetilde{\Delta}_{31}}{2} \\
& -2 s_{12}^{\prime} s_{13}^{\prime} c_{13}^{\prime 2} c_{23}\left(c_{12}^{\prime} s_{23} \cos \delta+s_{12}^{\prime} s_{13}^{\prime} c_{23}\right)\left(4 \sin ^{2} \frac{\widetilde{\Delta}_{21}}{2} \sin ^{2} \frac{\widetilde{\Delta}_{31}}{2}+\sin \widetilde{\Delta}_{21} \sin \widetilde{\Delta}_{31}\right) \\
& +4 s_{12}^{\prime} c_{12}^{\prime} s_{13}^{\prime} c_{13}^{\prime 2} s_{23} c_{23} \sin \delta\left(\sin ^{2} \frac{\widetilde{\Delta}_{21}}{2} \sin \widetilde{\Delta}_{31}-\sin ^{2} \frac{\widetilde{\Delta}_{31}}{2} \sin \widetilde{\Delta}_{21}\right) \\
& \stackrel{s_{12}^{\prime} \approx 1}{\longrightarrow} c_{23}^{2} \sin ^{2}\left(2 \theta_{13}^{\prime}\right) \sin ^{2} \frac{\widetilde{\Delta}_{32}}{2} \text {. }
\end{aligned}
$$

From figure 3 , it is clear that the factor $\sin ^{2}\left(2 \theta_{13}^{\prime}\right)$ in these expressions behaves quite differently depending on the mass hierarchy. For normal hierarchy $\sin ^{2}\left(2 \theta_{13}^{\prime}\right)$ will peak around $a \approx \delta m_{31}^{2}$ but for the inverted hierarchy case it will not. This will become manifest if the factor $\sin ^{2}\left(\widetilde{\Delta}_{32} / 2\right)$ also peaked at or near the same energy. ${ }^{5}$

For the normal hierarchy case, when $a \approx \delta m_{31}^{2}$ we have

$$
\delta \lambda_{32}=\lambda_{+}^{\prime \prime}-\lambda_{-}^{\prime \prime} \approx \sqrt{\left[\lambda_{+}^{\prime}-\left(\delta m_{31}^{2}+a s_{13}^{2}\right)\right]^{2}+4 a^{2} c_{13}^{2} s_{13}^{2}} \approx 2 s_{13} a
$$

\footnotetext{
${ }^{5}$ If we expand the running parameters in our eq. (4.4) in powers of the vacuum $s_{13}$ and $\alpha=\delta m_{21}^{2} / \delta m_{31}^{2}$, the leading order term expressed using the notation of Freund [22] takes the form

$$
P\left(\nu_{e} \rightarrow \nu_{\mu}\right)=s_{23}^{2} \sin ^{2}\left(2 \theta_{13}^{\prime}\right) \sin ^{2} \frac{\widetilde{\Delta}_{32}}{2}=s_{23}^{2} \frac{4 s_{13}^{2}}{(\hat{A}-1)^{2}} \sin ^{2}[(\hat{A}-1) \hat{\Delta}]+\cdots
$$

where $\hat{A}=a / \delta m_{31}^{2}$ and $\hat{\Delta}=\delta m_{31}^{2} L / 4 E$. This is the same as eq. (38a) of Freund with $\sin ^{2} 2 \theta_{13}$ replaced by $4 s_{13}^{2}$, and agrees with the corresponding term of Akhmedov et al. [29]. The enhancement discussed in the main text can be seen to occur at $\hat{A}=1$, that is $a=\delta m_{31}^{2}$, which is possible only when $\delta m_{31}^{2}>0$. However, the formulae of Cervera et al. [27], Akhmedov et al. [29], Asano and Minakata [32], and Freund [22] compared in the previous section all suffer in accuracy around the resonance region $\hat{A} \approx 1$. This is not the case for our expression, which has a smooth transition across the resonance. The fact that the CP-phase dependent terms are negligible at the relevant energies and baselines is also clear in our approach.
} 


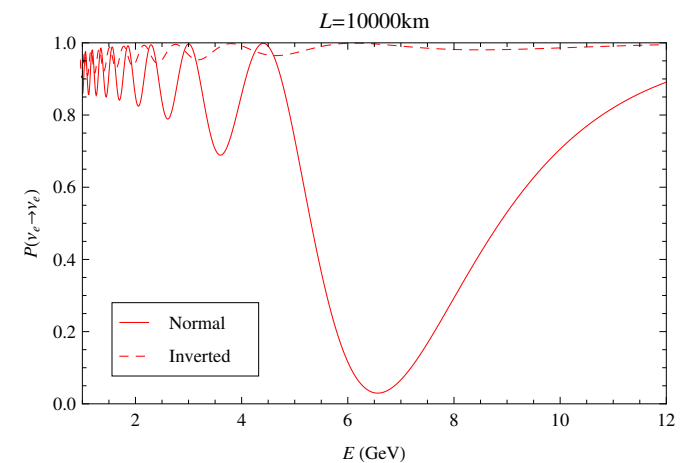

(a)

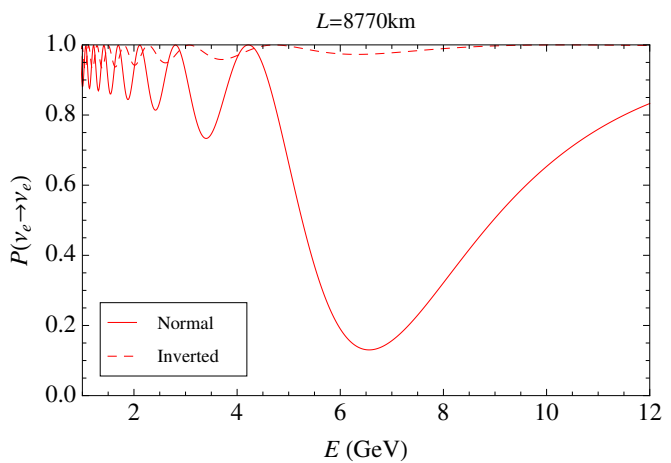

(b)

Figure 10. Comparison of the exact oscillation probabilities $P\left(\nu_{e} \rightarrow \nu_{e}\right)$ between the normal and inverted hierarchies at (a) $L=10000 \mathrm{~km}\left(\rho=4.53 \mathrm{~g} / \mathrm{cm}^{3}\right)$, and (b) $L=8770 \mathrm{~km}\left(\rho=4.33 \mathrm{~g} / \mathrm{cm}^{3}\right)$

Therefore,

$$
\begin{aligned}
\frac{\tilde{\Delta}_{32}}{2}=\frac{\delta \lambda_{32}}{4 E} L \approx \frac{s_{13} a}{2 E} L & =\left(2.9 \times 10^{-5}\right)\left(\frac{\rho}{\mathrm{g} / \mathrm{cm}^{3}}\right)\left(\frac{L}{\mathrm{~km}}\right) \\
& =\frac{\pi}{2}\left(\frac{\rho L}{54000\left(\mathrm{~km} \cdot \mathrm{g} / \mathrm{cm}^{3}\right)}\right) .
\end{aligned}
$$

From figure 1 , it is clear that $\rho L<54000\left(\mathrm{~km} \cdot \mathrm{g} / \mathrm{cm}^{3}\right)$ as long as the neutrino beam does not enter the core of the Earth, at which point the constant average matter density approximation breaks down. Therefore, in order to take $\widetilde{\Delta}_{32} / 2$ as close as possible to $\pi / 2$ while preventing the beam from entering the Earth's core, we need $L \sim 10000 \mathrm{~km}$.

For instance, if we take $L=10000 \mathrm{~km}$ for which $\rho=4.53 \mathrm{~g} / \mathrm{cm}^{3}$, we have $\rho L \approx$ $45300 \mathrm{~km} \cdot \mathrm{g} / \mathrm{cm}^{3}$. The value of $\widetilde{\Delta}_{32} / 2$ at resonance $a \approx \delta m_{31}^{2}$ is then

$$
\frac{\pi}{2} \times \frac{45300}{54000}=0.42 \pi
$$

leading to an oscillation peak/dip factor of $\sin ^{2}\left(\widetilde{\Delta}_{32} / 2\right)=0.94$. Using eq. (2.2), the neutrino beam energy at which $a \approx \delta m_{31}^{2}$ is found to be

$$
\frac{E}{\mathrm{GeV}}=\frac{\left(\delta m_{31}^{2} / \mathrm{eV}^{2}\right)}{\left(7.63 \times 10^{-5}\right) \times\left(\rho /\left(\mathrm{g} / \mathrm{cm}^{3}\right)\right)}=\frac{\left(2.47 \times 10^{-3}\right)}{\left(7.63 \times 10^{-5}\right) \times(4.53)} \approx 7 .
$$

Indeed, in figure 10(a) we show the exact $\nu_{e}$ survival probabilities at $L=10000 \mathrm{~km}$ for both hierarchies, and we can see that the normal hierarchy case dips by over $95 \%$ around $E \sim 6.5 \mathrm{GeV}$. Thus, our rough estimates give a highly reliable result.

If we take a somewhat shorter baseline of $L=8770 \mathrm{~km}$, which is the distance between CERN and Kamioka [47], we have $\rho=4.33 \mathrm{~g} / \mathrm{cm}^{3}$, and $\rho L \approx 38000 \mathrm{~km} \cdot \mathrm{g} / \mathrm{cm}^{3}$. The value of $\widetilde{\Delta}_{32} / 2$ at resonance $a \approx \delta m_{31}^{2}$ is then

$$
\frac{\pi}{2} \times \frac{38000}{54000}=0.35 \pi
$$


leading to an oscillation peak/dip factor of $\sin ^{2}\left(\widetilde{\Delta}_{32} / 2\right)=0.8$, which is still fairly prominent. Using eq. (2.2), the neutrino beam energy at which $a \approx \delta m_{31}^{2}$ is found to be

$$
\frac{E}{\mathrm{GeV}}=\frac{\left(\delta m_{31}^{2} / \mathrm{eV}^{2}\right)}{\left(7.63 \times 10^{-5}\right) \times\left(\rho /\left(\mathrm{g} / \mathrm{cm}^{3}\right)\right)}=\frac{\left(2.47 \times 10^{-3}\right)}{\left(7.63 \times 10^{-5}\right) \times(4.33)} \approx 7.5 .
$$

The actual oscillation peak occurs slightly off resonance around $E=6.5 \mathrm{GeV}$ as can already be seen in figure 7. Comparison of $P\left(\nu_{e} \rightarrow \nu_{e}\right)$ at $L=8770 \mathrm{~km}$ with $\delta=0$ for the normal and inverted hierarchies are shown in figure 10(b). $P\left(\nu_{e} \rightarrow \nu_{\mu}\right)$ is compared in figure 11(b).

The differences between the normal and inverted hierarchies for both baselines is manifest, indicating that measuring these oscillation probabilities at this baseline would allow us to determine the mass hierarchy easily. (We consider the dependence on the $\mathrm{CP}$ violating phase $\delta$ in the next section.) Eqs. (4.1), (4.4), and (4.5) also suggest that the measurement may provide a better determination of $\sin ^{2} \theta_{23}$.

\subsection{The "magic" baseline}

The "magic" baseline is the baseline at which the dependence of $P\left(\nu_{e} \rightarrow \nu_{\mu}\right)$ on the CP violating phase $\delta$ vanishes [49]. ${ }^{6}$ Looking at eq. (4.2), the only term without $\delta$-dependence is the $\left|\tilde{U}_{e 3}\right|^{2}\left|\tilde{U}_{\mu 3}\right|^{2}$ term. To make every other term vanish, we must have

$$
\sin \frac{\widetilde{\Delta}_{21}}{2}=\sin \left(\frac{\delta \lambda_{21}}{4 E} L\right)=0
$$

Therefore, the magic baseline condition is

$$
\frac{\delta \lambda_{21}}{4 E} L=n \pi, \quad n \in \mathbb{Z} .
$$

If we are in the energy and mass-density range such that $\delta m_{21}^{2}<a<\left|\delta m_{31}^{2}\right|$, we can see from figure 4 that $\delta \lambda_{21} \approx a=2 \sqrt{2} G_{F} N_{e} E$, and the above condition reduces to

$$
\sqrt{2} G_{F} N_{e} L \approx 2 n \pi
$$

which is the usual magic baseline condition. Using eq. (2.2), this condition for the $n=1$ case becomes

$$
\frac{\widetilde{\Delta}_{21}}{2} \approx \frac{a}{4 E} L=\left(9.7 \times 10^{-5}\right)\left(\frac{\rho}{\mathrm{g} / \mathrm{cm}^{3}}\right)\left(\frac{L}{\mathrm{~km}}\right)=\pi
$$

that is

$$
\frac{\rho L}{\mathrm{~km} \cdot \mathrm{g} / \mathrm{cm}^{3}} \approx 32000 \text {. }
$$

This is satisfied for $L \approx 7500 \mathrm{~km}$ as can be read off of figure 1 . Indeed, in figure 11(a) we plot the bands that $P\left(\nu_{e} \rightarrow \nu_{\mu}\right)$ at $L=7500 \mathrm{~km}$ sweeps for both mass hierarchies when $\delta$ is varied throughout its range of $[0,2 \pi]$. We can see that the dependence on $\delta$ is very weak.

\footnotetext{
${ }^{6}$ An illuminating discussion on the physical meaning of the "magic baseline" can be found in ref. [50].
} 


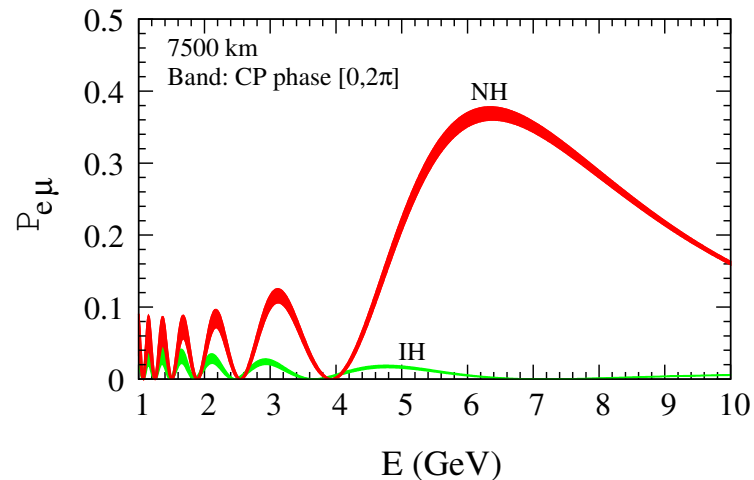

(a)

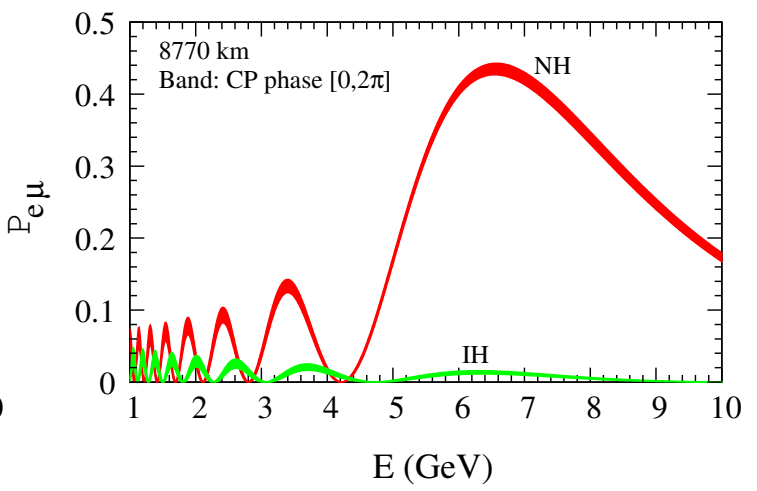

(b)

Figure 11. The dependence of the exact oscillation probability $P\left(\nu_{e} \rightarrow \nu_{\mu}\right)$ on the CP violating phase $\delta$ at (a) $L=7500 \mathrm{~km}\left(\rho=4.21 \mathrm{~g} / \mathrm{cm}^{3}\right)$, and (b) $L=8770 \mathrm{~km}\left(\rho=4.33 \mathrm{~g} / \mathrm{cm}^{3}\right)$ for the normal (red) and inverted (green) mass hierarchies.

However, if we look at eq. (4.3) carefully, it is clear that all terms that include the CP violating phase $\delta$ are multiplied by $c_{12}^{\prime}$ which goes to zero when $a \gg \delta m_{21}^{2}$. Indeed, this was why $\delta$ did not appear in eq. (4.4). The condition $a \gg \delta m_{21}^{2}$ demands

$$
\left(\frac{\rho}{\mathrm{g} / \mathrm{cm}^{3}}\right)\left(\frac{E}{\mathrm{GeV}}\right) \gg 1,
$$

which is clearly satisfied around the oscillation peak for the $L=8770 \mathrm{~km}$ case just discussed in the previous section. Thus, $P\left(\nu_{e} \rightarrow \nu_{\mu}\right)$ for this baseline is also only very weakly dependent on $\delta$ as shown in figure 11(b). We can conclude that, in general, as long as eq. (4.17) is satisfied, one does not need to be at a specific "magic" baseline to suppress the $\delta$-dependence of $P\left(\nu_{e} \rightarrow \nu_{\mu}\right)$.

\section{Summary}

We have presented a new and simple approximation for calculating the neutrino oscillation matter effects. Our approximation was derived utilizing the Jacobi method [44], and we show in the appendix that at most two rotations are sufficient for approximate diagonalization of the effective Hamiltonian. The two rotation angles are absorbed into effective values of $\theta_{12}$ and $\theta_{13}$.

As explained in detail in the appendix, the approximation works when $\theta_{13}=O(\varepsilon)$, where $\varepsilon=\sqrt{\delta m_{21}^{2} /\left|\delta m_{31}^{2}\right|}=0.17$, a condition which has been shown to be satisfied by Daya Bay [33] and RENO [35]. Our formulae are compact and can easily be coded as well as be manipulated by hand. The application of our method to finding the $\nu_{e} \rightarrow \nu_{\mu}, \nu_{\tau}$ resonance conversion condition, and that to the determination of the 'magic' baseline $[49,50]$ have been demonstrated.

In this paper, only the matter effect due to Standard Model $W$ exchange between the neutrinos and matter was considered. New Physics effects which distinguish between 
neutrino flavor would add extra terms to the effective Hamiltonian, which would require further rotations for diagonalization. This has been discussed previously in ref. [43], and the potential constraints on New Physics from long baseline neutrino oscillations experiments in refs. [51-53]. Updates to these works will be presented in future publications.

\section{Acknowledgments}

We would like to thank Minako Honda and Naotoshi Okamura for their contributions to the earlier version of this work [42, 43]. Helpful communications with W. Liao, T. Ohlsson, S. Petcov, P. Roy, and K. Takeuchi are gratefully acknowledged. SKA was supported by the DST/INSPIRE Research Grant [IFA-PH-12], Department of Science \& Technology, India. SKA is also grateful for the support of IFIC-CSIC, University of Valencia, Spain, where some initial portions of this work was carried out. TT was supported by the U.S. Department of Energy, grant number DE-FG05-92ER40677 task A, and by the World Premier International Research Center Initiative (WPI Initiative), MEXT, Japan.

\section{A Conventions, notation, and basic formulae}

Here, we collect the basic formulae associated with neutrino oscillation in order to fix our notation and conventions.

\section{A.1 The PMNS matrix}

Assuming three-generation neutrino mixing, the flavor eigenstates $\left|\nu_{\alpha}\right\rangle(\alpha=e, \mu, \tau)$ are related to the three mass eigenstates $\left|\nu_{j}\right\rangle(j=1,2,3)$ via the Pontecorvo-Maki-NakagawaSakata (PMNS) matrix [54-56]

$$
\left(V_{\mathrm{PMNS}}\right)_{\alpha j} \equiv\left\langle\nu_{\alpha} \mid \nu_{j}\right\rangle
$$

that is,

$$
\begin{aligned}
& \left|\nu_{j}\right\rangle=\sum_{\alpha=e, \mu, \tau}\left|\nu_{\alpha}\right\rangle\left\langle\nu_{\alpha} \mid \nu_{j}\right\rangle=\sum_{\alpha=e, \mu, \tau}\left(V_{\mathrm{PMNS}}\right)_{\alpha j}\left|\nu_{\alpha}\right\rangle, \\
& \left|\nu_{\alpha}\right\rangle=\sum_{j=1,2,3}\left|\nu_{j}\right\rangle\left\langle\nu_{j} \mid \nu_{\alpha}\right\rangle=\sum_{j=1,2,3}\left(V_{\mathrm{PMNS}}\right)_{\alpha j}^{*}\left|\nu_{j}\right\rangle .
\end{aligned}
$$

The standard parametrization is given by

$$
V_{\mathrm{PMNS}}=U \mathcal{P}
$$

with $^{7}$

$$
\begin{aligned}
U & =R_{23}\left(\theta_{23}, 0\right) R_{13}\left(\theta_{13}, \delta\right) R_{12}\left(\theta_{12}, 0\right) \\
& =\left[\begin{array}{ccc}
1 & 0 & 0 \\
0 & c_{23} & s_{23} \\
0 & -s_{23} & c_{23}
\end{array}\right]\left[\begin{array}{ccc}
c_{13} & 0 & s_{13} e^{-i \delta} \\
0 & 1 & 0 \\
-s_{13} e^{i \delta} & 0 & c_{13}
\end{array}\right]\left[\begin{array}{ccc}
c_{12} & s_{12} & 0 \\
-s_{12} & c_{12} & 0 \\
0 & 0 & 1
\end{array}\right]
\end{aligned}
$$

\footnotetext{
${ }^{7}$ Cervera et al. in ref. [27] use a different convention in which the sign of $\delta$ is flipped.
} 


$$
\begin{aligned}
= & {\left[\begin{array}{ccc}
c_{12} c_{13} & s_{12} c_{13} & s_{13} e^{-i \delta} \\
-s_{12} c_{23}-c_{12} s_{13} s_{23} e^{i \delta} & c_{12} c_{23}-s_{12} s_{13} s_{23} e^{i \delta} & c_{13} s_{23} \\
s_{12} s_{23}-c_{12} s_{13} c_{23} e^{i \delta} & -c_{12} s_{23}-s_{12} s_{13} c_{23} e^{i \delta} & c_{13} c_{23}
\end{array}\right], } \\
\mathcal{P} & =\operatorname{diag}\left(1, e^{i \alpha_{21} / 2}, e^{i \alpha_{31} / 2}\right) .
\end{aligned}
$$

Here, $R_{i j}(\theta, \delta)$ denotes a rotation matrix in the $i j$-plane of clockwise rotation angle $\theta$ with phases $\pm \delta$ on the off-diagonal $j i$ and $i j$-elements, respectively, and $s_{i j} \equiv \sin \theta_{i j}$, $c_{i j} \equiv \cos \theta_{i j}$. Without loss of generality, we can adopt the convention $0 \leq \theta_{i j} \leq \pi / 2$, $0 \leq \delta<2 \pi$ [57]. Of the six parameters in this expression and the three neutrino masses, which add up to a total of nine parameters, neutrino $\rightarrow$ neutrino oscillations are only sensitive to six:

- the three mixing angles: $\theta_{12}, \theta_{23}, \theta_{13}$,

- two mass-squared differences: $\delta m_{21}^{2}, \delta m_{31}^{2}$, where $\delta m_{i j}^{2}=m_{i}^{2}-m_{j}^{2}$, and

- the CP-violating phase: $\delta$.

The Majorana phases, $\alpha_{21}$ and $\alpha_{31}$, only appear in lepton-number violating processes such as neutrinoless double beta decay, and cannot be determined via neutrino $\rightarrow$ neutrino oscillations. The absolute scale of the neutrino masses also remain undetermined since neutrino oscillation is an interference effect.

\section{A.2 Neutrino oscillation}

If a neutrino of flavor $\alpha$ is created at $x=0$ with energy $E$, then the state of the neutrino at $x=0$ is

$$
\left|\nu_{\alpha, 0}(x=0)\right\rangle=\left|\nu_{\alpha}\right\rangle=\sum_{j=1}^{3}\left(V_{\mathrm{PMNS}}\right)_{\alpha j}^{*}\left|\nu_{j}\right\rangle
$$

At $x=L$, the same state is

$$
\left|\nu_{\alpha, 0}(x=L)\right\rangle=\sum_{j=1}^{3} e^{i p_{j} L}\left(V_{\mathrm{PMNS}}\right)_{\alpha j}^{*}\left|\nu_{j}\right\rangle=e^{i p_{1} L} \sum_{j=1}^{3} e^{i\left(p_{j}-p_{1}\right) L}\left(V_{\mathrm{PMNS}}\right)_{\alpha j}^{*}\left|\nu_{j}\right\rangle .
$$

Assing $m_{j} \ll E$ we can approximate

$$
p_{j}=\sqrt{E^{2}-m_{j}^{2}}=E-\frac{m_{j}^{2}}{2 E}+\cdots
$$

so that

$$
p_{j}-p_{1} \approx-\frac{\delta m_{j 1}^{2}}{2 E}, \quad \delta m_{j 1}^{2}=m_{j}^{2}-m_{1}^{2}
$$

and we find

$$
\left|\nu_{\alpha, 0}(x=L)\right\rangle=e^{i p_{1} L} \sum_{j=1}^{3} \exp \left(-i \frac{\delta m_{j 1}^{2}}{2 E} L\right)\left(V_{\mathrm{PMNS}}\right)_{\alpha j}^{*}\left|\nu_{j}\right\rangle
$$


Therefore, the amplitude of observing the neutrino of flavor $\beta$ at $x=L$ is given by (dropping the irrelevant overall phase)

$$
\begin{aligned}
\mathcal{A}_{\beta \alpha} & =\left\langle\nu_{\beta} \mid \nu_{\alpha, 0}(x=L)\right\rangle \\
& =\left[\sum_{k=1}^{3}\left\langle\nu_{k}\right|\left(V_{\mathrm{PMNS}}\right)_{\beta k}\right]\left[\sum_{j=1}^{3} \exp \left(-i \frac{\delta m_{j 1}^{2}}{2 E} L\right)\left(V_{\mathrm{PMNS}}\right)_{\alpha j}^{*}\left|\nu_{j}\right\rangle\right] \\
& =\sum_{j=1}^{3}\left(V_{\mathrm{PMNS}}\right)_{\beta j} \exp \left(-i \frac{\delta m_{j 1}^{2}}{2 E} L\right)\left(V_{\mathrm{PMNS}}\right)_{\alpha j}^{*} \\
& =\sum_{j=1}^{3} U_{\beta j} \exp \left(-i \frac{\delta m_{j 1}^{2}}{2 E} L\right) U_{\alpha j}^{*} \\
& =\left[U \exp \left(-i \frac{\delta M^{2}}{2 E} L\right) U^{\dagger}\right]_{\beta \alpha} \\
& =\left[\exp \left(-i \frac{H_{0}}{2 E} L\right)\right]_{\beta \alpha},
\end{aligned}
$$

where

$$
\delta M^{2}=\left[\begin{array}{ccc}
0 & 0 & 0 \\
0 & \delta m_{21}^{2} & 0 \\
0 & 0 & \delta m_{31}^{2}
\end{array}\right]
$$

and

$$
H_{0}=U \delta M^{2} U^{\dagger}
$$

Thus, the probability of oscillation from $\left|\nu_{\alpha}\right\rangle$ to $\left|\nu_{\beta}\right\rangle$ with neutrino energy $E$ and baseline $L$ is given by

$$
\begin{aligned}
P\left(\nu_{\alpha} \rightarrow \nu_{\beta}\right) & =\left|\mathcal{A}_{\beta \alpha}\right|^{2} \\
& =\left|\sum_{j=1}^{3} U_{\beta j} \exp \left(-i \frac{\delta m_{j 1}^{2}}{2 E} L\right) U_{\alpha j}^{*}\right|^{2} \\
& =\delta_{\alpha \beta}-4 \sum_{i>j} \Re\left(U_{\alpha i}^{*} U_{\beta i} U_{\alpha j} U_{\beta j}^{*}\right) \sin ^{2} \frac{\Delta_{i j}}{2}+2 \sum_{i>j} \Im\left(U_{\alpha i}^{*} U_{\beta i} U_{\alpha j} U_{\beta j}^{*}\right) \sin \Delta_{i j},
\end{aligned}
$$

where $^{8}$

$$
\Delta_{i j} \equiv \frac{\delta m_{i j}^{2}}{2 E} L=2.534\left(\frac{\delta m_{i j}^{2}}{\mathrm{eV}^{2}}\right)\left(\frac{\mathrm{GeV}}{E}\right)\left(\frac{L}{\mathrm{~km}}\right), \quad \delta m_{i j}^{2} \equiv m_{i}^{2}-m_{j}^{2} .
$$

Since

$$
\Delta_{32}=\Delta_{31}-\Delta_{21},
$$

\footnotetext{
${ }^{8}$ Note that our notation differs from that of Cervera et al. in ref. [27]. There, the symbol $\Delta_{i j}$ is defined without the factor of $L$, that is, $\Delta_{i j}=\delta m_{i j}^{2} / 2 E$. It also differs from that used by Freund in ref. [22] where $\Delta=\delta m_{31}^{2}$, and $\hat{\Delta}=\delta m_{31}^{2} L / 4 E$. Huber and Winter in ref. [49] define $\Delta=\delta m_{31}^{2} L / 4 E$, which is also used in ref. [58]. So care is necessary when comparing formulae.
} 
only two of the three $\Delta_{i j}$ 's in eq. (A.13) are independent. Eliminating $\Delta_{32}$ from eq. (A.13) for the $\alpha=\beta$ case yields

$$
\begin{aligned}
P\left(\nu_{\alpha} \rightarrow \nu_{\alpha}\right)=1 & -4\left|U_{\alpha 2}\right|^{2}\left(1-\left|U_{\alpha 2}\right|^{2}\right) \sin ^{2} \frac{\Delta_{21}}{2}-4\left|U_{\alpha 3}\right|^{2}\left(1-\left|U_{\alpha 3}\right|^{2}\right) \sin ^{2} \frac{\Delta_{31}}{2} \\
& +2\left|U_{\alpha 2}\right|^{2}\left|U_{\alpha 3}\right|^{2}\left(4 \sin ^{2} \frac{\Delta_{21}}{2} \sin ^{2} \frac{\Delta_{31}}{2}+\sin \Delta_{21} \sin \Delta_{31}\right),
\end{aligned}
$$

and for the $\alpha \neq \beta$ case we have

$$
\begin{aligned}
P\left(\nu_{\alpha} \rightarrow \nu_{\beta}\right)= & 4\left|U_{\alpha 2}\right|^{2}\left|U_{\beta 2}\right|^{2} \sin ^{2} \frac{\Delta_{21}}{2}+4\left|U_{\alpha 3}\right|^{2}\left|U_{\beta 3}\right|^{2} \sin ^{2} \frac{\Delta_{31}}{2} \\
& +2 \Re\left(U_{\alpha 3}^{*} U_{\beta 3} U_{\alpha 2} U_{\beta 2}^{*}\right)\left(4 \sin ^{2} \frac{\Delta_{21}}{2} \sin ^{2} \frac{\Delta_{31}}{2}+\sin \Delta_{21} \sin \Delta_{31}\right) \\
& +4 J_{(\alpha, \beta)}\left(\sin ^{2} \frac{\Delta_{21}}{2} \sin \Delta_{31}-\sin ^{2} \frac{\Delta_{31}}{2} \sin \Delta_{21}\right),
\end{aligned}
$$

where $J_{(\alpha, \beta)}$ is the Jarlskog invariant [59]:

$$
\begin{aligned}
J_{(\alpha, \beta)} & =+\Im\left(U_{\alpha 1}^{*} U_{\beta 1} U_{\alpha 2} U_{\beta 2}^{*}\right)=+\Im\left(U_{\alpha 2}^{*} U_{\beta 2} U_{\alpha 3} U_{\beta 3}^{*}\right)=+\Im\left(U_{\alpha 3}^{*} U_{\beta 3} U_{\alpha 1} U_{\beta 1}^{*}\right) \\
& =-\Im\left(U_{\alpha 2}^{*} U_{\beta 2} U_{\alpha 1} U_{\beta 1}^{*}\right)=-\Im\left(U_{\alpha 1}^{*} U_{\beta 1} U_{\alpha 3} U_{\beta 3}^{*}\right)=-\Im\left(U_{\alpha 3}^{*} U_{\beta 3} U_{\alpha 2} U_{\beta 2}^{*}\right) \\
& =-J_{(\beta, \alpha)} .
\end{aligned}
$$

In the parametrization given in eq. (A.4), we have

$$
J_{(\mu, e)}=-J_{(e, \mu)}=J_{(e, \tau)}=-J_{(\tau, e)}=J_{(\tau, \mu)}=-J_{(\mu, \tau)}=\hat{J} \sin \delta,
$$

with

$$
\hat{J}=s_{12} c_{12} s_{13} c_{13}^{2} s_{23} c_{23} .
$$

The oscillation probabilities for the anti-neutrinos are obtained by replacing $U_{\alpha i}$ with its complex conjugate, which only amounts to flipping the sign of $\delta$ in the parametrization of eq. (A.4). It is clear from eq. (A.16) that $P\left(\bar{\nu}_{\alpha} \rightarrow \bar{\nu}_{\alpha}\right)=P\left(\nu_{\alpha} \rightarrow \nu_{\alpha}\right)$, which is to be expected from the CPT theorem. For flavor changing oscillations, only the Jarskog term in eq. (A.17) changes sign.

\section{A.3 Matter effects}

If the matter density along the baseline is constant, matter effects on neutrino oscillations can be taken into account by replacing the PMNS matrix elements and mass-squared differences with their "effective" values in matter:

$$
\Delta_{i j} \rightarrow \widetilde{\Delta}_{i j}, \quad U_{\alpha i} \rightarrow \tilde{U}_{\alpha i}
$$

where $\tilde{U}$ is the unitary matrix that diagonalizes the modified Hamiltonian,

$$
H_{a}=\tilde{U}\left[\begin{array}{ccc}
\lambda_{1} & 0 & 0 \\
0 & \lambda_{2} & 0 \\
0 & 0 & \lambda_{3}
\end{array}\right] \tilde{U}^{\dagger}=\underbrace{U\left[\begin{array}{ccc}
0 & 0 & 0 \\
0 & \delta m_{21}^{2} & 0 \\
0 & 0 & \delta m_{31}^{2}
\end{array}\right]}_{=\delta M^{2}} U_{=H_{0}}^{\dagger}+\left[\begin{array}{lll}
a & 0 & 0 \\
0 & 0 & 0 \\
0 & 0 & 0
\end{array}\right],
$$


and

$$
\widetilde{\Delta}_{i j}=\frac{\delta \lambda_{i j}}{2 E} L, \quad \delta \lambda_{i j}=\lambda_{i}-\lambda_{j}
$$

The factor $a$ is due to the interaction of the $\left|\nu_{e}\right\rangle$ component of the neutrinos with the electrons in matter via $W$-exchange:

$$
a=2 \sqrt{2} G_{F} N_{e} E .
$$

Assuming $N_{e}=N_{p} \approx N_{n}$ in Earth matter, $N_{e}$ for mass density per unit volume of $\rho$ can be expressed using Avogadro's number $N_{A}=6.02214129 \times 10^{23} \mathrm{~mol}^{-1}$ as

$$
N_{e}=N_{p} \approx \rho N_{A} / 2=\left(3.011 \times 10^{23} / \mathrm{cm}^{3}\right) \times\left(\frac{\rho}{\mathrm{g} / \mathrm{cm}^{3}}\right) .
$$

Thus, putting back powers of $\hbar c$ to convert from natural to conventional units, we find

$$
\begin{aligned}
a & =2 \sqrt{2} G_{F} N_{e} E \times(\hbar c)^{3} \\
& =\left(7.63 \times 10^{-5} \mathrm{eV}^{2}\right)\left(\frac{\rho}{\mathrm{g} / \mathrm{cm}^{3}}\right)\left(\frac{E}{\mathrm{GeV}}\right) .
\end{aligned}
$$

For anti-neutrino beams, $a$ is replaced by $-a$ in eq. (A.22). Note that $a$ is $E$-dependent, which means that both $\widetilde{U}$ and $\widetilde{\Delta}_{i j}$ are also $E$-dependent. It is also assumed that $E \ll M_{W}$ since the $W$-exchange interaction is approximated by a point-like four-fermion interaction in deriving this expression.

\section{B Jacobi method}

\section{B.1 Setup}

As mentioned in the introduction, it is possible to write down exact analytical expressions for $\widetilde{\Delta}_{i j}$ and $\tilde{U}_{\alpha i}[16]$. However, simpler and more transparent approximate expressions can be obtained using the Jacobi method as will be shown in the following.

We introduce the matrix

$$
\mathcal{Q}=\operatorname{diag}\left(1,1, e^{i \delta}\right),
$$

and start with the partially diagonalized Hamiltonian:

$$
\begin{aligned}
H_{a}^{\prime} & =\mathcal{Q}^{\dagger} U^{\dagger} H_{a} U \mathcal{Q} \\
& =\mathcal{Q}^{\dagger}\left\{\left[\begin{array}{ccc}
0 & 0 & 0 \\
0 & \delta m_{21}^{2} & 0 \\
0 & 0 & \delta m_{31}^{2}
\end{array}\right]+U^{\dagger}\left[\begin{array}{lll}
a & 0 & 0 \\
0 & 0 & 0 \\
0 & 0 & 0
\end{array}\right] U\right\} \mathcal{Q} \\
& =\mathcal{Q}^{\dagger}\left[\begin{array}{ccc}
0 & 0 & 0 \\
0 & \delta m_{21}^{2} & 0 \\
0 & 0 & \delta m_{31}^{2}
\end{array}\right] \mathcal{Q}+a \mathcal{Q}^{\dagger}\left[\begin{array}{ccc}
U_{e 1}^{*} U_{e 1} & U_{e 1}^{*} U_{e 2} & U_{e 1}^{*} U_{e 3} \\
U_{e 2}^{*} U_{e 1} & U_{e 2}^{*} U_{e 2} & U_{e 2}^{*} U_{e 3} \\
U_{e 3}^{*} U_{e 1} & U_{e 3}^{*} U_{e 2} & U_{e 3}^{*} U_{e 3}
\end{array}\right] \mathcal{Q} \\
& =\left[\begin{array}{ccc}
0 & 0 & 0 \\
0 & \delta m_{21}^{2} & 0 \\
0 & 0 & \delta m_{31}^{2}
\end{array}\right]+a\left[\begin{array}{ccc}
c_{12}^{2} c_{13}^{2} & c_{12} s_{12} c_{13}^{2} & c_{12} c_{13} s_{13} \\
c_{12} s_{12} c_{13}^{2} & s_{12}^{2} c_{13}^{2} & s_{12} c_{13} s_{13} \\
c_{12} c_{13} s_{13} & s_{12} c_{13} s_{13} & s_{13}^{2}
\end{array}\right]
\end{aligned}
$$




$$
=\left[\begin{array}{ccc}
a c_{12}^{2} c_{13}^{2} & a c_{12} s_{12} c_{13}^{2} & a c_{12} c_{13} s_{13} \\
a c_{12} s_{12} c_{13}^{2} & a s_{12}^{2} c_{13}^{2}+\delta m_{21}^{2} & a s_{12} c_{13} s_{13} \\
a c_{12} c_{13} s_{13} & a s_{12} c_{13} s_{13} & a s_{13}^{2}+\delta m_{31}^{2}
\end{array}\right] .
$$

The matrix $\mathcal{Q}$ serves to rid $H_{a}^{\prime}$ of any reference to the $\mathrm{CP}$ violating phase $\delta$. The strategy we used in our previous papers $[42,43]$ was to approximately diagonalize $H_{a}^{\prime}$ through the Jacobi method using

$$
\varepsilon=\sqrt{\frac{\delta m_{21}^{2}}{\left|\delta m_{31}^{2}\right|}} \approx 0.17
$$

as the parameter to keep track of the sizes of the off-diagonal elements. We argued that approximate diagonalization was achieved when the off-diagonal elements were of order $O\left(\varepsilon^{2} s_{13}\left|\delta m_{31}^{2}\right|\right)$.

Note that our $\varepsilon$ differs from Asano and Minakata's $\epsilon$ in ref. [32] where

$$
\epsilon=\frac{\delta m_{21}^{2}}{\left|\delta m_{31}^{2}\right|} \approx 0.03
$$

That is, $\epsilon=\varepsilon^{2}$. So care is necessary when comparing formulae.

\section{B.2 Diagonalization of a $2 \times 2$ matrix}

Recall that for $2 \times 2$ real symmetric matrices, such as

$$
M=\left[\begin{array}{ll}
\alpha & \beta \\
\beta & \gamma
\end{array}\right], \quad \alpha, \beta, \gamma \in \mathbb{R}
$$

diagonalization is trivial. Just define

$$
R=\left[\begin{array}{rr}
c_{\omega} & s_{\omega} \\
-s_{\omega} & c_{\omega}
\end{array}\right], \quad \text { where } \quad c_{\omega}=\cos \omega, \quad s_{\omega}=\sin \omega, \quad \tan 2 \omega \equiv \frac{2 \beta}{\gamma-\alpha},
$$

and we obtain

$$
R^{\dagger} M R=\left[\begin{array}{cc}
\Lambda_{1} & 0 \\
0 & \Lambda_{2}
\end{array}\right]
$$

with

$$
\begin{aligned}
& \Lambda_{1}=\frac{\alpha c_{\omega}^{2}-\gamma s_{\omega}^{2}}{c_{\omega}^{2}-s_{\omega}^{2}}=\frac{(\alpha+\gamma) \mp \sqrt{(\alpha-\gamma)^{2}+4 \beta^{2}}}{2}, \\
& \Lambda_{2}=\frac{\gamma c_{\omega}^{2}-\alpha s_{\omega}^{2}}{c_{\omega}^{2}-s_{\omega}^{2}}=\frac{(\alpha+\gamma) \pm \sqrt{(\alpha-\gamma)^{2}+4 \beta^{2}}}{2}
\end{aligned}
$$

where the upper and lower signs are for the cases $\alpha<\gamma$ and $\alpha>\gamma$, respectively. The Jacobi method [44] entails iteratively diagonalizing $2 \times 2$ submatrices of a larger matrix in the order that requires the largest rotation angle at each step. In the limit of infinite iterations of this procedure, the matrix will converge to a diagonal matrix.

In the case of $H_{a}^{\prime}$ given in eq. (B.2), at most two iterations are sufficient to achieve approximate diagonalization, neglecting off-diagonal elements of order $O\left(\varepsilon^{2} s_{13}\left|\delta m_{31}^{2}\right|\right)$, regardless of the size of $a$. We demonstrate this in this appendix. 


\section{B.3 Neutrino case}

\section{B.3.1 Mixing angles and mass-squared differences}

Let us first evaluate the sizes of the sines and cosines of the three vacuum mixing angles in comparison to the parameter $\varepsilon$ defined in eq. (B.3). The current best fit values for the mass-squared differences and mixing angles are listed in table 1 . The sines and cosines of the central values of the mixing angles are

$$
\begin{array}{ll}
s_{23}=0.71, & c_{23}=0.71, \\
s_{12}=0.55, & c_{12}=0.84, \\
s_{13}=0.15, & c_{13}=0.99 .
\end{array}
$$

Therefore, $s_{13}$ is $O(\varepsilon)$ while all other sines and cosines are $O(1)$.

\section{B.3.2 First rotation}

The effective hamiltonian we need to diagonalize is

$$
\begin{aligned}
H_{a}^{\prime} & =\left[\begin{array}{ccc}
a c_{12}^{2} c_{13}^{2} & a c_{12} s_{12} c_{13}^{2} & a c_{12} c_{13} s_{13} \\
a c_{12} s_{12} c_{13}^{2} & a s_{12}^{2} c_{13}^{2}+\delta m_{21}^{2} & a s_{12} c_{13} s_{13} \\
a c_{12} c_{13} s_{13} & a s_{12} c_{13} s_{13} & a s_{13}^{2}+\delta m_{31}^{2}
\end{array}\right] \\
& =\left[\begin{array}{ccc}
a \mathcal{O}(1) & a \mathcal{O}(1) & a \mathcal{O}(\varepsilon) \\
a \mathcal{O}(1) & a \mathcal{O}(1)+\delta m_{21}^{2} & a \mathcal{O}(\varepsilon) \\
a \mathcal{O}(\varepsilon) & a \mathcal{O}(\varepsilon) & a \mathcal{O}\left(\varepsilon^{2}\right)+\delta m_{31}^{2}
\end{array}\right]
\end{aligned}
$$

Of the off-diagonal elements, the 1-2 element is the largest regardless of the size of $a$. Therefore, our first step is to diagonalize the 1-2 submatrix.

Define

$$
V \equiv\left[\begin{array}{ccc}
c_{\varphi} & s_{\varphi} & 0 \\
-s_{\varphi} & c_{\varphi} & 0 \\
0 & 0 & 1
\end{array}\right]
$$

where

$$
c_{\varphi}=\cos \varphi, \quad s_{\varphi}=\sin \varphi, \quad \tan 2 \varphi \equiv \frac{a c_{13}^{2} \sin 2 \theta_{12}}{\delta m_{21}^{2}-a c_{13}^{2} \cos 2 \theta_{12}}, \quad\left(0 \leq \varphi \leq \frac{\pi}{2}\right) .
$$

Using $V$, we find

$$
H_{a}^{\prime \prime} \equiv V^{\dagger} H_{a}^{\prime} V=\left[\begin{array}{ccc}
\lambda_{-}^{\prime} & 0 & a c_{12}^{\prime} c_{13} s_{13} \\
0 & \lambda_{+}^{\prime} & a s_{12}^{\prime} c_{13} s_{13} \\
a c_{12}^{\prime} c_{13} s_{13} & a s_{12}^{\prime} c_{13} s_{13} & a s_{13}^{2}+\delta m_{31}^{2}
\end{array}\right],
$$

where

$$
c_{12}^{\prime}=\cos \theta_{12}^{\prime}, \quad s_{12}^{\prime}=\sin \theta_{12}^{\prime}, \quad \theta_{12}^{\prime} \equiv \theta_{12}+\varphi,
$$

and

$$
\lambda_{ \pm}^{\prime} \equiv \frac{\left(\delta m_{21}^{2}+a c_{13}^{2}\right) \pm \sqrt{\left(\delta m_{21}^{2}-a c_{13}^{2}\right)^{2}+4 a c_{13}^{2} s_{12}^{2} \delta m_{21}^{2}}}{2}
$$




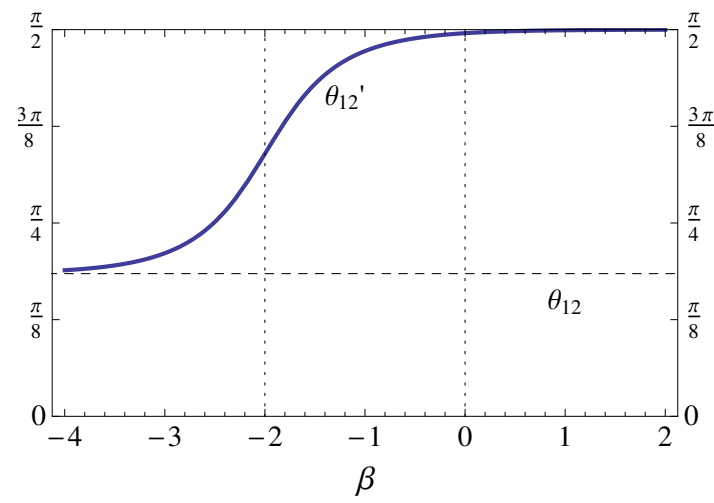

(a)

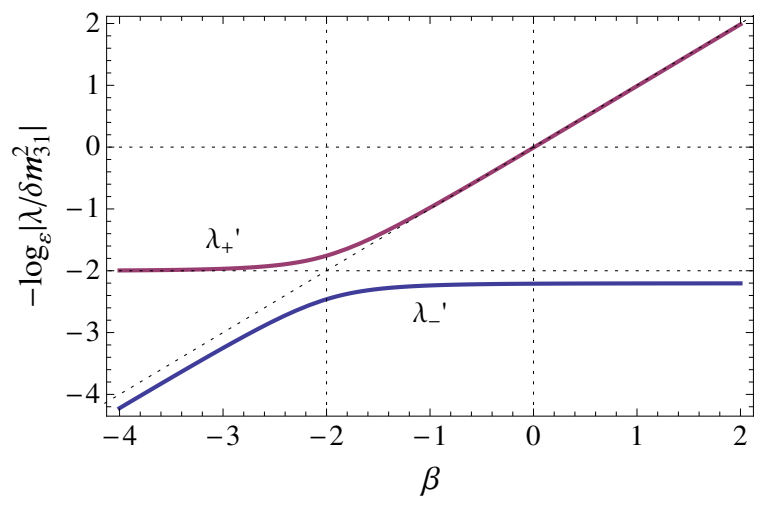

(b)

Figure 12. (a) The dependence of $\theta_{12}^{\prime}$ on $\beta=-\log _{\varepsilon}\left(a /\left|\delta m_{31}^{2}\right|\right)$. (b) The $\beta$-dependence of $\lambda_{ \pm}^{\prime}$.

The angle $\theta_{12}^{\prime}=\theta_{12}+\varphi$ can be calculated directly without calculating $\varphi$ via

$$
\tan 2 \theta_{12}^{\prime}=\frac{\delta m_{21}^{2} \sin 2 \theta_{12}}{\delta m_{21}^{2} \cos 2 \theta_{12}-a c_{13}^{2}}, \quad\left(\theta_{12} \leq \theta_{12}^{\prime} \leq \frac{\pi}{2}\right)
$$

The dependences of $\theta_{12}^{\prime}$ and $\lambda_{ \pm}^{\prime}$ on $\beta=-\log _{\varepsilon}\left(a /\left|\delta m_{31}^{2}\right|\right)$ are plotted in figure 12. Note that $\theta_{12}^{\prime}$ increases monotonically from $\theta_{12}$ to $\pi / 2$ with increasing $a$. The $\beta$-dependence of $s_{12}^{\prime}=\sin \theta_{12}^{\prime}$ and $c_{12}^{\prime}=\cos \theta_{12}^{\prime}$ are shown in figure $13(\mathrm{a})$. For $a \gg \delta m_{21}^{2}, s_{12}^{\prime}$ and $c_{12}^{\prime}$ behave as

$$
\begin{aligned}
& s_{12}^{\prime}=1-\frac{s_{12}^{2} c_{12}^{2}}{2}\left(\frac{\delta m_{21}^{2}}{a c_{13}^{2}}\right)^{2}+\cdots, \\
& c_{12}^{\prime}=s_{12} c_{12}\left(\frac{\delta m_{21}^{2}}{a c_{13}^{2}}\right)+s_{12} c_{12}\left(c_{12}^{2}-s_{12}^{2}\right)\left(\frac{\delta m_{21}^{2}}{a c_{13}^{2}}\right)^{2}+\cdots .
\end{aligned}
$$

Therefore, for $a \gg \delta m_{21}^{2}$ we have $a s_{12}^{\prime} \approx a$ while $a c_{12}^{\prime} \approx \delta m_{21}^{2} s_{12} c_{12} / c_{13}^{2}=$ $\varepsilon^{2}\left|\delta m_{31}^{2}\right| s_{12} c_{12} / c_{13}^{2} \approx 0.014\left|\delta m_{31}^{2}\right|=O\left(\varepsilon^{2}\left|\delta m_{31}^{2}\right|\right)$. This behavior is shown in figure 13(b). Note that $a c_{12}^{\prime}$ never grows larger than $O\left(\varepsilon^{2}\left|\delta m_{31}^{2}\right|\right)$ for any $a$.

The values of $\lambda_{ \pm}^{\prime}$ away from the level crossing point $a \sim \delta m_{21}^{2}$ for the $a \ll \delta m_{21}^{2}$ case are given by

$$
\begin{aligned}
& \lambda_{-}^{\prime}=a c_{13}^{2} c_{12}^{2}\left[1-s_{12}^{2}\left(\frac{a c_{13}^{2}}{\delta m_{21}^{2}}\right)-s_{12}^{2}\left(c_{12}^{2}-s_{12}^{2}\right)\left(\frac{a c_{13}^{2}}{\delta m_{21}^{2}}\right)^{2}+\cdots\right], \\
& \lambda_{+}^{\prime}=\delta m_{21}^{2}\left[1+s_{12}^{2}\left(\frac{a c_{13}^{2}}{\delta m_{21}^{2}}\right)+s_{12}^{2} c_{12}^{2}\left(\frac{a c_{13}^{2}}{\delta m_{21}^{2}}\right)^{2}+s_{12}^{2} c_{12}^{2}\left(c_{12}^{2}-s_{12}^{2}\right)\left(\frac{a c_{13}^{2}}{\delta m_{21}^{2}}\right)^{3}+\cdots\right],
\end{aligned}
$$




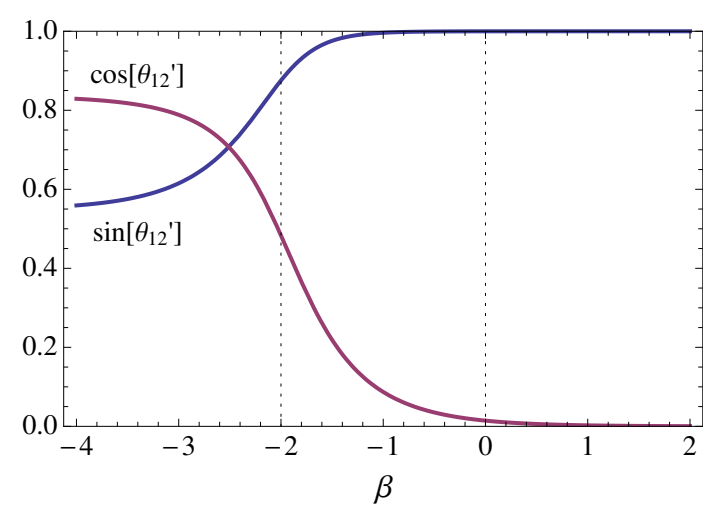

(a)

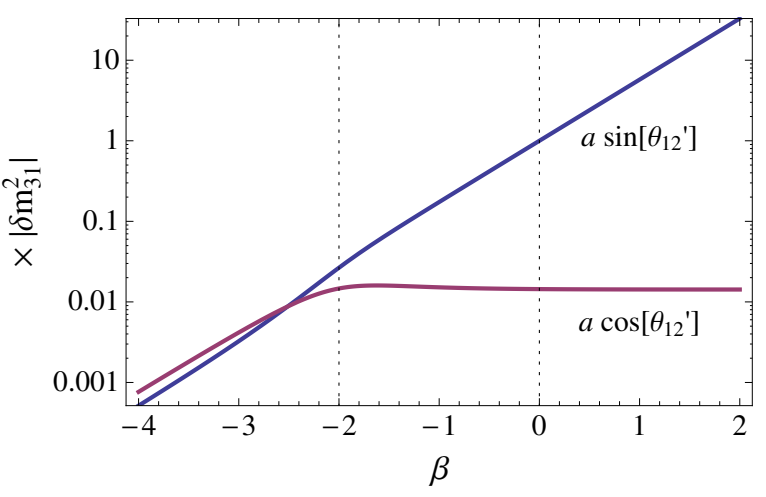

(b)

Figure 13. (a) The dependence of $s_{12}^{\prime}=\sin \theta_{12}^{\prime}$ and $c_{12}^{\prime}=\cos \theta_{12}^{\prime}$ on $\beta=-\log _{\varepsilon}\left(a /\left|\delta m_{31}^{2}\right|\right)$. (b) The dependence of $a s_{12}^{\prime}$ and $a c_{12}^{\prime}$ on $\beta$. The values are given in units of $\left|\delta m_{31}^{2}\right|$. The asymptotic value of $a c_{12}^{\prime}$ is $\delta m_{21}^{2} s_{12} c_{12} / c_{13}^{2} \approx 0.014\left|\delta m_{31}^{2}\right|=O\left(\varepsilon^{2}\left|\delta m_{31}^{2}\right|\right)$.

and those for the $a \gg \delta m_{21}^{2}$ case by

$$
\begin{aligned}
& \lambda_{-}^{\prime}=\delta m_{21}^{2} c_{12}^{2}\left[1-s_{12}^{2}\left(\frac{\delta m_{21}^{2}}{a c_{13}^{2}}\right)-s_{12}^{2}\left(c_{12}^{2}-s_{12}^{2}\right)\left(\frac{\delta m_{21}^{2}}{a c_{13}^{2}}\right)^{2}+\cdots\right] \\
& \lambda_{+}^{\prime}=a c_{13}^{2}\left[1+s_{12}^{2}\left(\frac{\delta m_{21}^{2}}{a c_{13}^{2}}\right)+s_{12}^{2} c_{12}^{2}\left(\frac{\delta m_{21}^{2}}{a c_{13}^{2}}\right)^{2}+s_{12}^{2} c_{12}^{2}\left(c_{12}^{2}-s_{12}^{2}\right)\left(\frac{\delta m_{21}^{2}}{a c_{13}^{2}}\right)^{3}+\cdots\right] .
\end{aligned}
$$

We will use this expansion for $\lambda_{+}^{\prime}$ later. Thus, the asymptotic values of $\lambda_{ \pm}^{\prime}$ are $\lambda_{-}^{\prime} \rightarrow$ $a c_{13}^{2} c_{12}^{2}, \lambda_{+}^{\prime} \rightarrow \delta m_{21}^{2}$ in the $a \rightarrow 0$ limit, and $\lambda_{-}^{\prime} \rightarrow \delta m_{21}^{2} c_{12}^{2}, \lambda_{+}^{\prime} \rightarrow a c_{13}^{2}$ in the $a \rightarrow \infty$ limit.

\section{B.3.3 Second rotation}

The effective hamiltonian after the first rotation was given by eq. (B.13). When $a<\delta m_{21}^{2}$, both non-zero off-diagonal elements are of order $O(\varepsilon a)<O\left(\varepsilon^{3}\left|\delta m_{31}^{2}\right|\right)$, since $s_{12}^{\prime}$ and $c_{12}^{\prime}$ are both $O(1)$ in that range as can be discerned from figure 13(a). However, as $a$ increases beyond $\delta m_{12}^{2}$ and $\theta_{12}^{\prime}$ approaches $\pi / 2$, we have $a s_{12}^{\prime} \rightarrow a, a c_{12}^{\prime} \rightarrow O\left(\varepsilon^{2}\left|\delta m_{31}^{2}\right|\right)$ and the 2-3 element becomes the larger of the two. Therefore, a 2-3 rotation is needed next.

We define

$$
W \equiv\left[\begin{array}{ccc}
1 & 0 & 0 \\
0 & c_{\phi} & s_{\phi} \\
0 & -s_{\phi} & c_{\phi}
\end{array}\right]
$$

where

$$
c_{\phi}=\cos \phi, \quad s_{\phi}=\sin \phi, \quad \tan 2 \phi \equiv \frac{a s_{12}^{\prime} \sin 2 \theta_{13}}{\delta m_{31}^{2}+a s_{13}^{2}-\lambda_{+}^{\prime}} .
$$




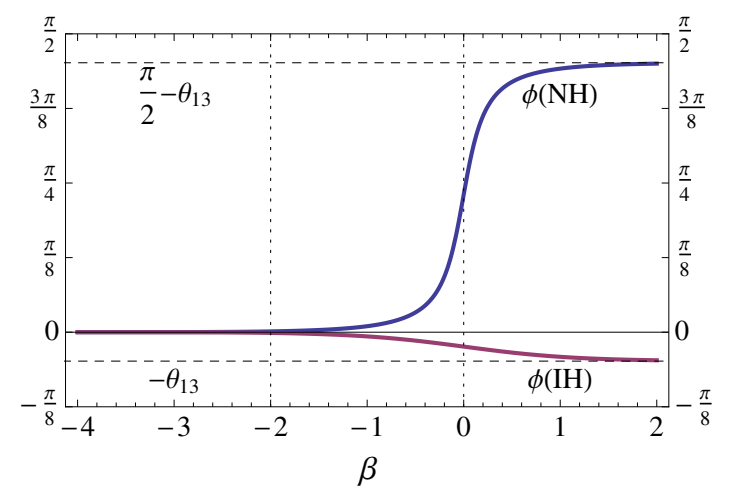

(a)

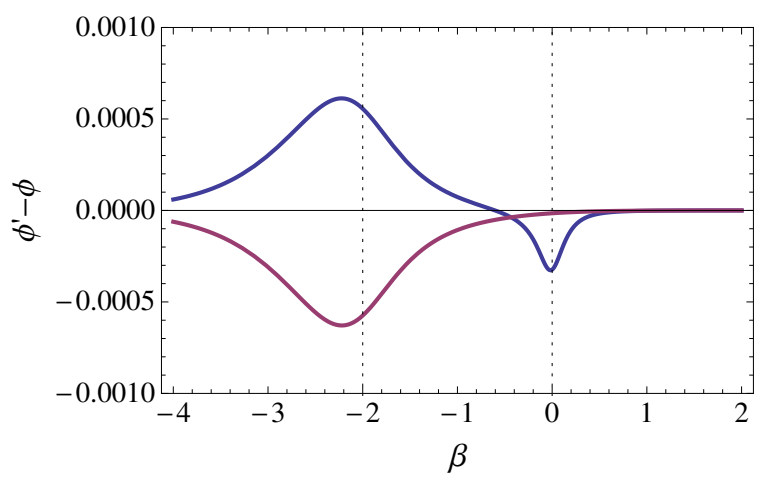

(b)

Figure 14. (a) The $\beta$-dependence of $\phi$ for the normal and inverted hierarchies. (b) The $\beta$ dependence of the difference $\phi^{\prime}-\phi$.

The angle $\phi$ is in the first quadrant when $\delta m_{31}^{2}>0$, and in the fourth quadrant when $\delta m_{31}^{2}<0$. Then,

$$
H_{a}^{\prime \prime \prime} \equiv W^{\dagger} H_{a}^{\prime \prime} W=\left[\begin{array}{ccc}
\lambda_{-}^{\prime} & -a c_{12}^{\prime} c_{13} s_{13} s_{\phi} & a c_{12}^{\prime} c_{13} s_{13} c_{\phi} \\
-a c_{12}^{\prime} c_{13} s_{13} s_{\phi} & \lambda_{\mp}^{\prime \prime} & 0 \\
a c_{12}^{\prime} c_{13} s_{13} c_{\phi} & 0 & \lambda_{ \pm}^{\prime \prime}
\end{array}\right]
$$

where the upper(lower) sign corresponds to the normal(inverted) hierarchy case with

$$
\lambda_{ \pm}^{\prime \prime} \equiv \frac{\left[\lambda_{+}^{\prime}+\left(\delta m_{31}^{2}+a s_{13}^{2}\right)\right] \pm \sqrt{\left[\lambda_{+}^{\prime}-\left(\delta m_{31}^{2}+a s_{13}^{2}\right)\right]^{2}+4 a^{2} s_{12}^{\prime 2} c_{13}^{2} s_{13}^{2}}}{2} .
$$

The $\beta$-dependences of $\lambda_{ \pm}^{\prime \prime}$ and $\phi$ are shown in figure $4((\mathrm{a})$ and (b)), and figure 14(a), respectively, for both mass hierarchies. For the normal hierarchy case, $\delta m_{31}^{2}>0$, the values of $\lambda_{ \pm}^{\prime \prime}$ away from the level crossing point $a \sim \delta m_{31}^{2}$ are approximately

$$
\begin{aligned}
& \lambda_{+}^{\prime \prime} \approx \delta m_{31}^{2}+a s_{13}^{2}, \\
& \lambda_{-}^{\prime \prime} \approx \lambda_{+}^{\prime},
\end{aligned}
$$

when $a \ll \delta m_{31}^{2}$, and

$$
\begin{aligned}
& \lambda_{+}^{\prime \prime} \approx a+s_{13}^{2} \delta m_{31}^{2}+c_{13}^{2} s_{12}^{2} \delta m_{21}^{2}, \\
& \lambda_{-}^{\prime \prime} \approx c_{13}^{2} \delta m_{31}^{2}+s_{13}^{2} s_{12}^{2} \delta m_{21}^{2},
\end{aligned}
$$

when $a \gg \delta m_{31}^{2}$. For the inverted hierarchy case, $\delta m_{31}^{2}<0$, where there is no level crossing, the values of $\lambda_{ \pm}^{\prime \prime}$ are approximately

$$
\lambda_{-}^{\prime \prime} \approx \delta m_{31}^{2}<0, \quad \lambda_{+}^{\prime \prime} \approx \lambda_{+}^{\prime}
$$

for all $a$. 
At this point, we argue that the angle $\phi$ defined in eq. (B.21) is well approximated by the angle $\phi^{\prime}$ which we define via

$$
\tan 2 \phi^{\prime} \equiv \frac{a \sin 2 \theta_{13}}{\left(\delta m_{31}^{2}-\delta m_{21}^{2} s_{12}^{2}\right)-a \cos 2 \theta_{13}} .
$$

This approximation is obtained by first noting that $\phi$ is significantly different from zero only when $a \gg \delta m_{21}^{2}$. The expansion of $\lambda_{+}^{\prime}$ in the denominator of the right-hand-side of eq. (B.21) in powers of $\delta m_{21}^{2} / a$ was given in eq. (B.19). Keeping only the first two terms, and noting also that $s_{12}^{\prime} \approx 1$ to the same order when $a \gg \delta m_{21}^{2}$ (cf. eq. (B.17)) we obtain eq. (B.27). The $\beta$-dependence of the difference $\phi^{\prime}-\phi$ is plotted in figure 14(b), and we can see that the disagreement is at most $O\left(\varepsilon^{4}\right)$. Thus, we replace $\phi$ with $\phi^{\prime}$ in the following.

Now, the effective Hamiltonian after the second rotation was given by eq. (B.22). Note that all of the non-zero off-diagonal elements include the factor $a c_{12}^{\prime}$, which is never larger than $O\left(\varepsilon^{2}\left|\delta m_{31}^{2}\right|\right)$ regardless of the value of $a$ as discussed above. They also all include a factor of $s_{13}$, which is $O(\varepsilon)$ as we have seen in eq. (B.9). Therefore, all off-diagonal elements of $H_{a}^{\prime \prime \prime}$ are of order $O\left(\varepsilon^{2} s_{13}\left|\delta m_{31}^{2}\right|\right)=O\left(\varepsilon^{3}\left|\delta m_{31}^{2}\right|\right)$ or smaller regardless of the size of $a$. Note that had the value of $s_{13}$ been smaller, the sizes of the neglected terms would have been proportionately smaller also. We conclude that, at this point, off-diagonal elements are negligible and a third rotation is not necessary.

\section{B.3.4 Absorption of $\phi^{\prime}$ into $\theta_{13}$}

From the above consideration, we conclude that the matrix which diagonalizes $H_{a}^{\prime}$, eq. (B.2), is given approximately by $V W$, and that the effective neutrino mixing matrix becomes

$$
\tilde{U} \approx U \mathcal{Q} V W=\underbrace{R_{23}\left(\theta_{23}, 0\right) R_{13}\left(\theta_{13}, \delta\right) R_{12}\left(\theta_{12}, 0\right)}_{U} \mathcal{Q} \underbrace{R_{12}(\varphi, 0)}_{V} \underbrace{R_{23}\left(\phi^{\prime}, 0\right)}_{W} .
$$

Using

$$
\begin{aligned}
& R_{12}\left(\theta_{12}, 0\right) \mathcal{Q}=\mathcal{Q} R_{12}\left(\theta_{12}, 0\right) \\
& R_{13}\left(\theta_{13}, \delta\right) \mathcal{Q}=\mathcal{Q} R_{13}\left(\theta_{13}, 0\right),
\end{aligned}
$$

we find

$$
\begin{aligned}
\tilde{U} & \approx R_{23}\left(\theta_{23}, 0\right) R_{13}\left(\theta_{13}, \delta\right) R_{12}\left(\theta_{12}, 0\right) \mathcal{Q} R_{12}(\varphi, 0) R_{23}\left(\phi^{\prime}, 0\right) \\
& =R_{23}\left(\theta_{23}, 0\right) \mathcal{Q} R_{13}\left(\theta_{13}, 0\right) R_{12}\left(\theta_{12}, 0\right) R_{12}(\varphi, 0) R_{23}\left(\phi^{\prime}, 0\right) \\
& =R_{23}\left(\theta_{23}, 0\right) \mathcal{Q} R_{13}\left(\theta_{13}, 0\right) R_{12}\left(\theta_{12}+\varphi, 0\right) R_{23}\left(\phi^{\prime}, 0\right) \\
& =R_{23}\left(\theta_{23}, 0\right) \mathcal{Q} R_{13}\left(\theta_{13}, 0\right) R_{12}\left(\theta_{12}^{\prime}, 0\right) R_{23}\left(\phi^{\prime}, 0\right) .
\end{aligned}
$$

Here, we argue that

$$
R_{12}\left(\theta_{12}^{\prime}, 0\right) R_{23}\left(\phi^{\prime}, 0\right) \approx R_{13}\left(\phi^{\prime}, 0\right) R_{12}\left(\theta_{12}^{\prime}, 0\right),
$$


that is, the $2-3$ rotation becomes a $1-3$ rotation when commuted through $R_{12}\left(\theta_{12}^{\prime}, 0\right)$. This is due to the fact that $\phi^{\prime}$ only becomes non-negligible when $a \gg \delta m_{12}^{2}$ where $s_{12}^{\prime} \approx 1$ and $c_{12}^{\prime} \approx 0$, which means

$$
R_{12}\left(\theta_{12}^{\prime}, 0\right) \approx\left[\begin{array}{ccc}
0 & 1 & 0 \\
-1 & 0 & 0 \\
0 & 0 & 1
\end{array}\right]
$$

and it is straightforward to see that

$$
\left[\begin{array}{ccc}
0 & 1 & 0 \\
-1 & 0 & 0 \\
0 & 0 & 1
\end{array}\right]\left[\begin{array}{ccc}
1 & 0 & 0 \\
0 & c_{\phi}^{\prime} & s_{\phi}^{\prime} \\
0 & -s_{\phi}^{\prime} & c_{\phi}^{\prime}
\end{array}\right]=\left[\begin{array}{ccc}
c_{\phi}^{\prime} & 0 & s_{\phi}^{\prime} \\
0 & 1 & 0 \\
-s_{\phi}^{\prime} & 0 & c_{\phi}^{\prime}
\end{array}\right]\left[\begin{array}{ccc}
0 & 1 & 0 \\
-1 & 0 & 0 \\
0 & 0 & 1
\end{array}\right],
$$

where $s_{\phi}^{\prime}=\sin \phi^{\prime}$ and $c_{\phi}^{\prime}=\cos \phi^{\prime}$. In the range $a \lesssim \delta m_{21}^{2}$, the angle $\phi^{\prime}$ is very small and both $R_{23}\left(\phi^{\prime}, 0\right)$ and $R_{13}\left(\phi^{\prime}, 0\right)$ are approximately unit matrices and eq. (B.31) is trivially satisfied. Curiously, this approximation breaks down around $a \sim \delta m_{31}^{2}$ for the normal hierarchy case when $\theta_{13}$ is $O\left(\varepsilon^{2}\right)$ or smaller, as is discussed in appendix C. However, given that the current experimentally preferred value of $\theta_{13}$ is $O(\varepsilon)$, the approximation is valid. Thus,

$$
\begin{aligned}
\tilde{U} & \approx R_{23}\left(\theta_{23}, 0\right) \mathcal{Q} R_{13}\left(\theta_{13}, 0\right) R_{12}\left(\theta_{12}^{\prime}, 0\right) R_{23}\left(\phi^{\prime}, 0\right) \\
& \approx R_{23}\left(\theta_{23}, 0\right) \mathcal{Q} R_{13}\left(\theta_{13}, 0\right) R_{13}\left(\phi^{\prime}, 0\right) R_{12}\left(\theta_{12}^{\prime}, 0\right) \\
& =R_{23}\left(\theta_{23}, 0\right) \mathcal{Q} R_{13}\left(\theta_{13}+\phi^{\prime}, 0\right) R_{12}\left(\theta_{12}^{\prime}, 0\right) \\
& =R_{23}\left(\theta_{23}, 0\right) \mathcal{Q} R_{13}\left(\theta_{13}^{\prime}, 0\right) R_{12}\left(\theta_{12}^{\prime}, 0\right) \\
& =R_{23}\left(\theta_{23}, 0\right) R_{13}\left(\theta_{13}^{\prime}, \delta\right) R_{12}\left(\theta_{12}^{\prime}, 0\right) \mathcal{Q},
\end{aligned}
$$

where we have defined

$$
\theta_{13}^{\prime} \equiv \theta_{13}+\phi^{\prime}
$$

This angle can be calculated directly without calculating $\phi^{\prime}$ via

$$
\tan 2 \theta_{13}^{\prime}=\frac{\left(\delta m_{31}^{2}-\delta m_{21}^{2} s_{12}^{2}\right) \sin 2 \theta_{13}}{\left(\delta m_{31}^{2}-\delta m_{21}^{2} s_{12}^{2}\right) \cos 2 \theta_{13}-a} .
$$

The diagonal phase matrix $\mathcal{Q}$ appearing rightmost in the above matrix product can be absorbed into the redefinition of the major phases and can be dropped. Thus, we arrive at our final approximation in which the vacuum mixing angles are replaced by their effective values in matter

$$
\begin{aligned}
\theta_{12} & \rightarrow \theta_{12}^{\prime}=\theta_{12}+\varphi, \\
\theta_{13} & \rightarrow \theta_{13}^{\prime}=\theta_{13}+\phi^{\prime}, \\
\theta_{23} & \rightarrow \theta_{23}, \\
\delta & \rightarrow \delta,
\end{aligned}
$$

and the eigenvalues of the effective Hamiltonian are given by

$$
\begin{aligned}
& \lambda_{1} \approx \lambda_{-}^{\prime}, \\
& \lambda_{2} \approx \lambda_{\mp}^{\prime \prime}, \\
& \lambda_{3} \approx \lambda_{ \pm}^{\prime \prime} .
\end{aligned}
$$

Note that of the mixing angles, only $\theta_{12}$ and $\theta_{13}$ are shifted. $\theta_{23}$ and $\delta$ stay at their vacuum values. 


\section{B.4 Anti-neutrino case}

\section{B.4.1 First rotation}

For the anti-neutrino case, the matter effect parameter $a$ acquires a minus sign. Thus, the effective hamiltonian to be diagonalized is

$$
\begin{aligned}
\bar{H}_{a}^{\prime} & =\left[\begin{array}{ccc}
-a c_{12}^{2} c_{13}^{2} & -a c_{12} s_{12} c_{13}^{2} & -a c_{12} c_{13} s_{13} \\
-a c_{12} s_{12} c_{13}^{2} & -a s_{12}^{2} c_{13}^{2}+\delta m_{21}^{2} & -a s_{12} c_{13} s_{13} \\
-a c_{12} c_{13} s_{13} & -a s_{12} c_{13} s_{13} & -a s_{13}^{2}+\delta m_{31}^{2}
\end{array}\right] \\
& =\left[\begin{array}{lll}
-a \mathcal{O}(1) & -a \mathcal{O}(1) & -a \mathcal{O}(\varepsilon) \\
-a \mathcal{O}(1) & -a \mathcal{O}(1)+\delta m_{21}^{2} & -a \mathcal{O}(\varepsilon) \\
-a \mathcal{O}(\varepsilon) & -a \mathcal{O}(\varepsilon) & -a \mathcal{O}\left(\varepsilon^{2}\right)+\delta m_{31}^{2}
\end{array}\right] .
\end{aligned}
$$

The largest off-diagonal element is the 1-2 element. Therefore, our first step is to diagonalize the 1-2 submatrix.

Define

$$
\bar{V} \equiv\left[\begin{array}{ccc}
\bar{c}_{\varphi} & \bar{s}_{\varphi} & 0 \\
-\bar{s}_{\varphi} & \bar{c}_{\varphi} & 0 \\
0 & 0 & 1
\end{array}\right]
$$

where

$$
\bar{c}_{\varphi}=\cos \bar{\varphi}, \quad \bar{s}_{\varphi}=\sin \bar{\varphi}, \quad \tan 2 \bar{\varphi} \equiv-\frac{a c_{13}^{2} \sin 2 \theta_{12}}{\delta m_{21}^{2}+a c_{13}^{2} \cos 2 \theta_{12}}, \quad\left(-\frac{\pi}{2}<\bar{\varphi}<0\right) .
$$

Using $\bar{V}$ we find

$$
\bar{H}_{a}^{\prime \prime} \equiv \bar{V}^{\dagger} \bar{H}_{a}^{\prime} \bar{V}=\left[\begin{array}{ccc}
\bar{\lambda}_{-}^{\prime} & 0 & -a \bar{c}_{12}^{\prime} c_{13} s_{13} \\
0 & \bar{\lambda}_{+}^{\prime} & -a \bar{s}_{12}^{\prime} c_{13} s_{13} \\
-a \bar{c}_{12}^{\prime} c_{13} s_{13} & -a \bar{s}_{12}^{\prime} c_{13} s_{13} & -a s_{13}^{2}+\delta m_{31}^{2}
\end{array}\right],
$$

where

$$
\bar{c}_{12}^{\prime}=\cos \bar{\theta}_{12}^{\prime}, \quad \bar{s}_{12}^{\prime}=\sin \bar{\theta}_{12}^{\prime}, \quad \bar{\theta}_{12}^{\prime} \equiv \theta_{12}+\bar{\varphi},
$$

and

$$
\bar{\lambda}_{ \pm}^{\prime} \equiv \frac{\left(\delta m_{21}^{2}-a c_{13}^{2}\right) \pm \sqrt{\left(\delta m_{21}^{2}+a c_{13}^{2}\right)^{2}-4 a c_{13}^{2} s_{12}^{2} \delta m_{21}^{2}}}{2} .
$$

The angle $\bar{\theta}_{12}^{\prime}$ can be calculated directly without going through $\bar{\varphi}$ via

$$
\tan 2 \bar{\theta}_{12}^{\prime}=\frac{\delta m_{21}^{2} \sin 2 \theta_{12}}{\delta m_{21}^{2} \cos 2 \theta_{12}+a c_{13}^{2}}, \quad\left(0 \leq \bar{\theta}_{12}^{\prime} \leq \theta_{12}\right) .
$$

The $\beta$-dependences of $\bar{\theta}_{12}^{\prime}$ and $\bar{\lambda}_{ \pm}^{\prime}$ are shown in figure 15 . Note that in contrast to the neutrino case, there is no level crossing. $\bar{\theta}_{12}^{\prime}$ decreases monotonically toward zero as $a$ is increased. For $a \gg \delta m_{21}^{2}, \bar{s}_{12}^{\prime}$ and $\bar{c}_{12}^{\prime}$ behave as

$$
\begin{aligned}
& \bar{s}_{12}^{\prime}=s_{12} c_{12}\left(\frac{\delta m_{21}^{2}}{a c_{13}^{2}}\right)-s_{12} c_{12}\left(c_{12}^{2}-s_{12}^{2}\right)\left(\frac{\delta m_{21}^{2}}{a c_{13}^{2}}\right)^{2}+\cdots, \\
& \bar{c}_{12}^{\prime}=1-\frac{s_{12}^{2} c_{12}^{2}}{2}\left(\frac{\delta m_{21}^{2}}{a c_{13}^{2}}\right)^{2}+\cdots,
\end{aligned}
$$




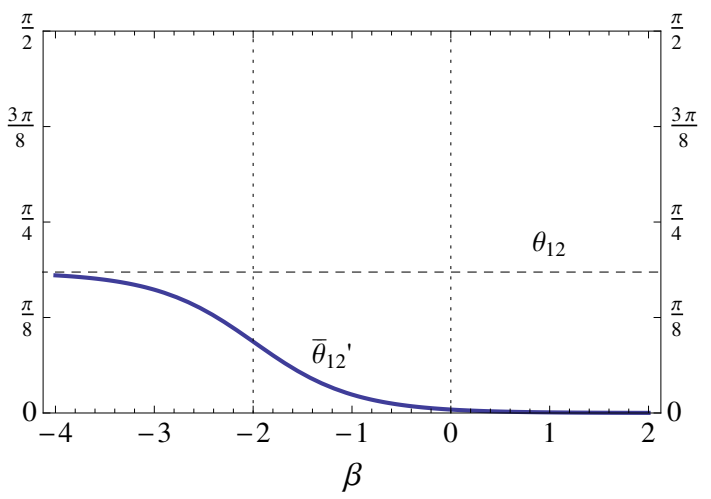

(a)

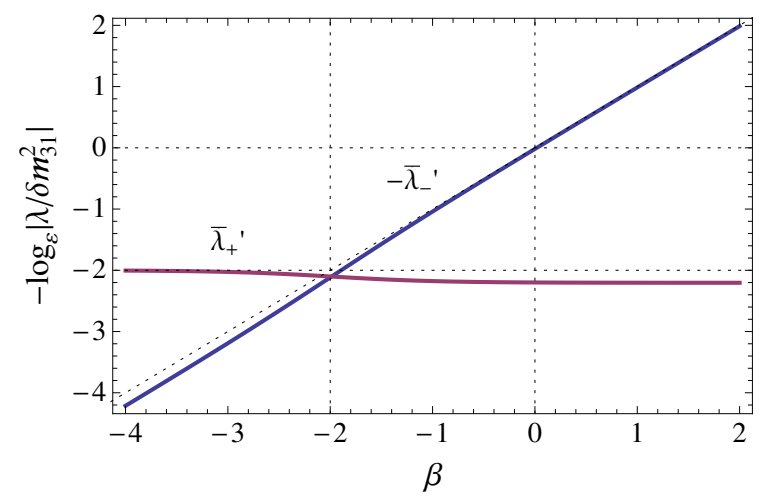

(b)

Figure 15. (a) The dependence of $\bar{\theta}_{12}^{\prime}$ on $\beta=-\log _{\varepsilon}\left(a /\left|\delta m_{31}^{2}\right|\right)$. (b) The $\beta$-dependence of $\bar{\lambda}_{ \pm}^{\prime}$.

and we see that, this time, we have $a \bar{c}_{12}^{\prime} \approx a$ and $a \bar{s}_{12}^{\prime} \approx \delta m_{21}^{2} s_{12} c_{12} / c_{13}^{2}=O\left(\varepsilon^{2}\left|\delta m_{31}^{2}\right|\right)$. These $\beta$-dependences of $\bar{s}_{12}^{\prime}, \bar{c}_{12}^{\prime}, a \bar{s}_{12}^{\prime}$, and $a \bar{c}_{12}^{\prime}$ are shown in figure 16(a) and (b).

In the range $a \ll \delta m_{21}^{2}, \bar{\lambda}_{ \pm}^{\prime}$ can be expanded as

$$
\begin{aligned}
& \bar{\lambda}_{-}^{\prime}=-a c_{13}^{2} c_{12}^{2}\left[1+s_{12}^{2}\left(\frac{a c_{13}^{2}}{\delta m_{21}^{2}}\right)-s_{12}^{2}\left(c_{12}^{2}-s_{12}^{2}\right)\left(\frac{a c_{13}^{2}}{\delta m_{21}^{2}}\right)^{2}+\cdots\right] \\
& \bar{\lambda}_{+}^{\prime}=\delta m_{21}^{2}\left[1-s_{12}^{2}\left(\frac{a c_{13}^{2}}{\delta m_{21}^{2}}\right)+s_{12}^{2} c_{12}^{2}\left(\frac{a c_{13}^{2}}{\delta m_{21}^{2}}\right)^{2}-s_{12}^{2} c_{12}^{2}\left(c_{12}^{2}-s_{12}^{2}\right)\left(\frac{a c_{13}^{2}}{\delta m_{21}^{2}}\right)^{3}+\cdots\right]
\end{aligned}
$$

while in the range $a \gg \delta m_{21}^{2}$, we obtain

$$
\begin{aligned}
& \bar{\lambda}_{-}^{\prime}=-a c_{13}^{2}\left[1-s_{12}^{2}\left(\frac{\delta m_{21}^{2}}{a c_{13}^{2}}\right)+s_{12}^{2} c_{12}^{2}\left(\frac{\delta m_{21}^{2}}{a c_{13}^{2}}\right)^{2}-s_{12}^{2} c_{12}^{2}\left(\frac{\delta m_{21}^{2}}{a c_{13}^{2}}\right)^{3}+\cdots\right], \\
& \bar{\lambda}_{+}^{\prime}=\delta m_{21}^{2} c_{12}^{2}\left[1+s_{12}^{2}\left(\frac{\delta m_{21}^{2}}{a c_{13}^{2}}\right)-s_{12}^{2}\left(c_{12}^{2}-s_{12}^{2}\right)\left(\frac{\delta m_{21}^{2}}{a c_{13}^{2}}\right)^{2}+\cdots\right] .
\end{aligned}
$$

The asymptotic values are thus $\bar{\lambda}_{+}^{\prime} \rightarrow \delta m_{21}^{2}, \bar{\lambda}_{-}^{\prime} \rightarrow-a c_{13}^{2} c_{12}^{2}$, in the limit $a \rightarrow 0$, and $\bar{\lambda}_{+}^{\prime} \rightarrow c_{12}^{2} \delta m_{21}^{2}, \bar{\lambda}_{-}^{\prime} \rightarrow-a c_{13}^{2}$, in the limit $a \rightarrow \infty$.

\section{B.4.2 Second rotation}

After the first rotation, the effective hamiltonian was given by eq. (B.42). When $a<\delta m_{21}^{2}$, both non-zero off-diagonal elements are of order $O(\varepsilon a)<O\left(\varepsilon^{3}\left|\delta m_{31}^{2}\right|\right)$. In contrast to the neutrino case, as $a$ increases beyond $\delta m_{21}^{2}$, the angle $\bar{\theta}_{12}^{\prime}$ approaches 0 , and it is the $1-3$ element that becomes the larger of the two. Therefore, a 1-3 rotation is needed next.

We define

$$
\bar{W}=\left[\begin{array}{ccc}
\bar{c}_{\phi} & 0 & \bar{s}_{\phi} \\
0 & 1 & 0 \\
-\bar{s}_{\phi} & 0 & \bar{c}_{\phi}
\end{array}\right],
$$




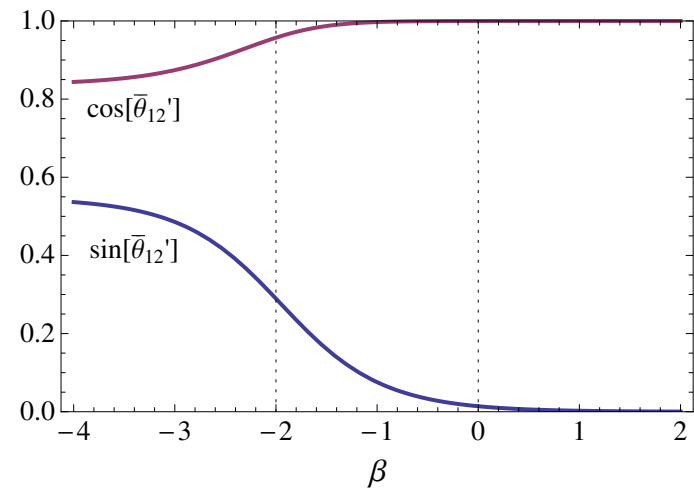

(a)

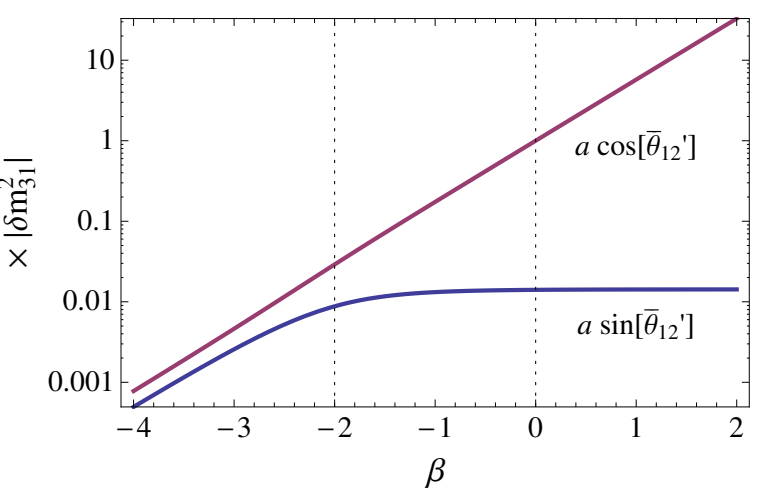

(b)

Figure 16. (a) The $\beta$-dependence of $\bar{s}_{12}^{\prime}=\sin \bar{\theta}_{12}^{\prime}$ and $\bar{c}_{12}^{\prime}=\cos \bar{\theta}_{12}^{\prime}$. (b) The $\beta$-dependence of $a \bar{s}_{12}^{\prime}$ and $a \bar{c}_{12}^{\prime}$. The asymptotic value of $a \bar{s}_{12}^{\prime}$ is $\delta m_{21}^{2} s_{12} c_{12} / c_{13}^{2} \approx 0.014\left|\delta m_{31}^{2}\right|=O\left(\varepsilon^{2}\left|\delta m_{31}^{2}\right|\right)$.

where

$$
\bar{c}_{\phi}=\cos \bar{\phi}, \quad \bar{s}_{\phi}=\cos \bar{\phi}, \quad \tan 2 \bar{\phi} \equiv-\frac{a \bar{c}_{12}^{\prime} \sin 2 \theta_{13}}{\delta m_{31}^{2}-a s_{13}^{2}-\bar{\lambda}_{-}^{\prime}} .
$$

The angle $\bar{\phi}$ is in the fourth quadrant when $\delta m_{31}^{2}>0$, and the first quadrant when $\delta m_{31}^{2}<$ 0 . Using $\bar{W}$, we find

$$
\bar{H}_{a}^{\prime \prime \prime} \equiv \bar{W}^{\dagger} \bar{H}_{a}^{\prime \prime} \bar{W}=\left[\begin{array}{ccc}
\bar{\lambda}_{\mp}^{\prime \prime} & a \bar{s}_{12}^{\prime} c_{13} s_{13} \bar{s}_{\phi} & 0 \\
a \bar{s}_{12}^{\prime} c_{13} s_{13} \bar{s}_{\phi} & \bar{\lambda}_{+}^{\prime} & -a \bar{s}_{12}^{\prime} c_{13} s_{13} \bar{c}_{\phi} \\
0 & -a \bar{s}_{12}^{\prime} c_{13} s_{13} \bar{c}_{\phi} & \bar{\lambda}_{ \pm}^{\prime \prime}
\end{array}\right],
$$

where the upper(lower) sign corresponds to normal(inverted) mass hierarchy with

$$
\bar{\lambda}_{ \pm}^{\prime \prime} \equiv \frac{\left[\bar{\lambda}_{-}^{\prime}+\left(\delta m_{31}^{2}-a s_{13}^{2}\right)\right] \pm \sqrt{\left[\bar{\lambda}_{-}^{\prime}-\left(\delta m_{31}^{2}-a s_{13}^{2}\right)\right]^{2}+4 a^{2} \bar{c}_{12}^{\prime 2} c_{13}^{2} s_{13}^{2}}}{2} .
$$

The $\beta$-dependence of $\bar{\lambda}_{ \pm}^{\prime \prime}$ and $\bar{\phi}$ are shown in figure $4((\mathrm{c})$ and $(\mathrm{d}))$, and figure $17(\mathrm{a})$, respectively, for both normal and inverted mass hierarchies. For the normal hierarchy case, $\delta m_{31}^{2}>0$, there is no level crossing, and $\bar{\lambda}_{ \pm}^{\prime \prime}$ are well approximated by

$$
\bar{\lambda}_{+}^{\prime \prime} \approx \delta m_{31}^{2}, \quad \bar{\lambda}_{-}^{\prime \prime} \approx \bar{\lambda}_{-}^{\prime} .
$$

Level crossing occurs for the inverted hierarchy case, $\delta m_{31}^{2}<0$, in which we have

$$
\begin{aligned}
& \bar{\lambda}_{+}^{\prime \prime} \approx \bar{\lambda}_{-}^{\prime}, \\
& \bar{\lambda}_{-}^{\prime \prime} \approx-\delta m_{31}^{2}-a s_{13}^{2},
\end{aligned}
$$

when $a \ll \delta m_{31}^{2}$, and

$$
\begin{aligned}
& \bar{\lambda}_{+}^{\prime \prime} \approx-c_{13}^{2} \delta m_{31}^{2}+s_{13}^{2} s_{12}^{2} \delta m_{21}^{2} \\
& \bar{\lambda}_{-}^{\prime \prime} \approx-a-s_{13}^{2} \delta m_{31}^{2}+c_{13}^{2} s_{12}^{2} \delta m_{21}^{2}
\end{aligned}
$$

when $a \gg \delta m_{31}^{2}$. 


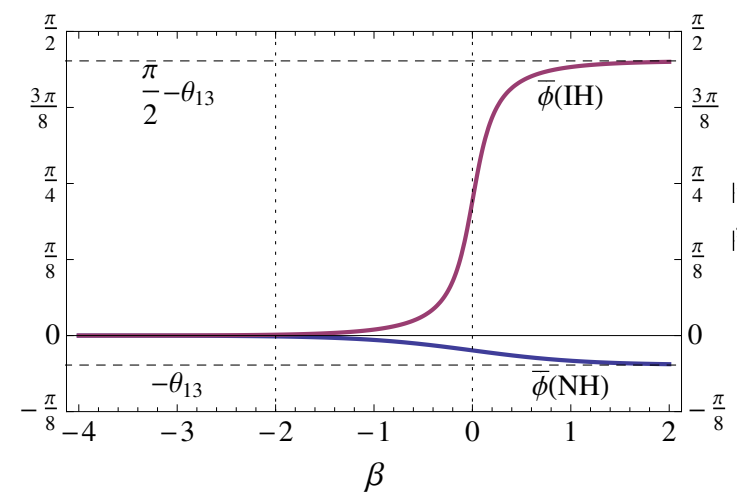

(a)

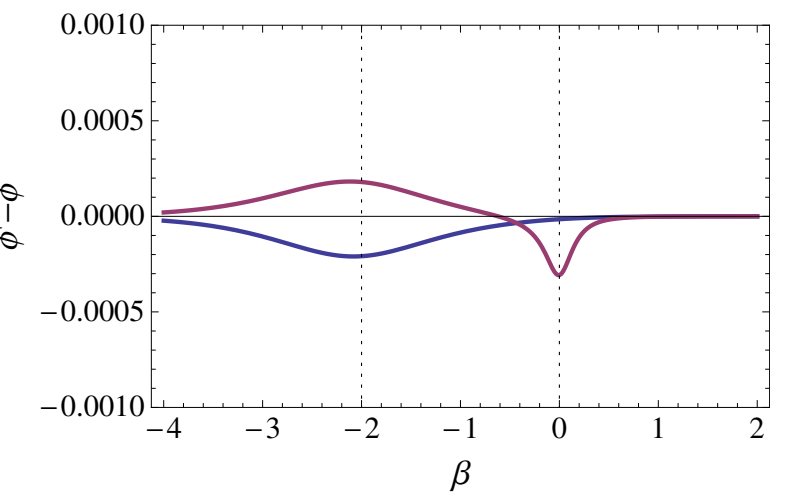

(b)

Figure 17. (a) The $\beta$-dependence of $\bar{\phi}$ for the normal and inverted hierarchies. (b) The $\beta$ dependence of the difference $\bar{\phi}^{\prime}-\bar{\phi}$.

Here, as in the neutrino case, we approximate $\bar{\phi}$ with the angle $\bar{\phi}^{\prime}$ defined via

$$
\tan 2 \bar{\phi}^{\prime}=-\frac{a \sin 2 \theta_{13}}{\left(\delta m_{31}^{2}-s_{12}^{2} \delta m_{21}^{2}\right)+a \cos ^{2} 2 \theta_{13}},
$$

which is obtained by using eqs. (B.46) and (B.48) on eq. (B.50). The difference between $\bar{\phi}^{\prime}$ and $\bar{\phi}$ is shown in figure $17(\mathrm{~b})$, and it is clear that the difference is negligible.

Now, the effective Hamiltonian after the second rotation was given by eq. (B.51). Note that all of the non-zero off-diagonal elements include the factor $a \bar{s}_{12}^{\prime}$, which is never larger than $O\left(\varepsilon^{2}\left|\delta m_{31}^{2}\right|\right)$ regardless of the value of $a$ as discussed above. They also all include a factor of $s_{13}$, which is $O(\varepsilon)$ as we have seen in eq. (B.9). Therefore, all off-diagonal elements of $\bar{H}_{a}^{\prime \prime \prime}$ are of order $O\left(\varepsilon^{2} s_{13}\left|\delta m_{31}^{2}\right|\right)=O\left(\varepsilon^{3}\left|\delta m_{31}^{2}\right|\right)$ or smaller regardless of the size of $a$. We conclude that, at this point, off-diagonal elements are negligible and a third rotation is not necessary.

\section{B.4.3 Absorption of $\bar{\phi}^{\prime}$ into $\theta_{13}$}

From the above consideration, we conclude that the matrix which diagonalizes $\bar{H}_{a}^{\prime}$, eq. (B.39), is given approximately by $\overline{V W}$, and that the effective anti-neutrino mixing matrix becomes

$$
\tilde{U} \approx U \mathcal{Q} \overline{V W}=\underbrace{R_{23}\left(\theta_{23}, 0\right) R_{13}\left(\theta_{13}, \delta\right) R_{12}\left(\theta_{12}, 0\right)}_{U} \mathcal{Q} \underbrace{R_{12}(\overline{\varphi, 0})}_{\bar{V}} \underbrace{R_{13}\left(\bar{\phi}^{\prime}, 0\right)}_{\bar{W}} .
$$

As in the neutrino case, we find

$$
\begin{aligned}
\widetilde{U} & \approx R_{23}\left(\theta_{23}, 0\right) R_{13}\left(\theta_{13}, \delta\right) R_{12}\left(\theta_{12}, 0\right) \mathcal{Q} R_{12}(\bar{\varphi}, 0) R_{13}\left(\bar{\phi}^{\prime}, 0\right) \\
& =R_{23}\left(\theta_{23}, 0\right) \mathcal{Q} R_{13}\left(\theta_{13}, 0\right) R_{12}\left(\theta_{12}, 0\right) R_{12}(\bar{\varphi}, 0) R_{13}\left(\bar{\phi}^{\prime}, 0\right) \\
& =R_{23}\left(\theta_{23}, 0\right) \mathcal{Q} R_{13}\left(\theta_{13}, 0\right) R_{12}\left(\theta_{12}+\bar{\varphi}, 0\right) R_{13}\left(\bar{\phi}^{\prime}, 0\right) \\
& =R_{23}\left(\theta_{23}, 0\right) \mathcal{Q} R_{13}\left(\theta_{13}, 0\right) R_{12}\left(\bar{\theta}_{12}^{\prime}, 0\right) R_{13}\left(\bar{\phi}^{\prime}, 0\right) .
\end{aligned}
$$


Here, we argue that

$$
R_{12}\left(\bar{\theta}_{12}^{\prime}, 0\right) R_{13}\left(\bar{\phi}^{\prime}, 0\right) \approx R_{13}\left(\bar{\phi}^{\prime}, 0\right) R_{12}\left(\bar{\theta}_{12}^{\prime}, 0\right),
$$

that is, the 1-3 rotation passes through $R_{12}\left(\bar{\theta}_{12}^{\prime}, 0\right)$. This is due to the fact that $\bar{\phi}^{\prime}$ only becomes non-negligible when $a \gg \delta m_{12}^{2}$ where $\bar{s}_{12}^{\prime} \approx 0$ and $\bar{c}_{12}^{\prime} \approx 1$, which means

$$
R_{12}\left(\bar{\theta}_{12}^{\prime}, 0\right) \approx\left[\begin{array}{lll}
1 & 0 & 0 \\
0 & 1 & 0 \\
0 & 0 & 1
\end{array}\right] \text {, }
$$

thus any matrix will commute with $R_{12}\left(\bar{\theta}_{12}^{\prime}, 0\right)$. In the range $a \lesssim \delta m_{21}^{2}$, the angle $\phi^{\prime}$ is very small and both $R_{23}\left(\phi^{\prime}, 0\right)$ and $R_{13}\left(\phi^{\prime}, 0\right)$ are approximately unit matrices and eq. (B.59) is trivially satisfied. The accuracy of this approximation is discussed in appendix C. Therefore,

$$
\begin{aligned}
\tilde{U} & \approx R_{23}\left(\theta_{23}, 0\right) \mathcal{Q} R_{13}\left(\theta_{13}, 0\right) R_{12}\left(\bar{\theta}_{12}^{\prime}, 0\right) R_{13}\left(\bar{\phi}^{\prime}, 0\right) \\
& \approx R_{23}\left(\theta_{23}, 0\right) \mathcal{Q} R_{13}\left(\theta_{13}, 0\right) R_{13}\left(\bar{\phi}^{\prime}, 0\right) R_{12}\left(\bar{\theta}_{12}^{\prime}, 0\right) \\
& =R_{23}\left(\theta_{23}, 0\right) \mathcal{Q} R_{13}\left(\theta_{13}+\bar{\phi}^{\prime}, 0\right) R_{12}\left(\bar{\theta}_{12}^{\prime}, 0\right) \\
& =R_{23}\left(\theta_{23}, 0\right) \mathcal{Q} R_{13}\left(\bar{\theta}_{13}^{\prime}, 0\right) R_{12}\left(\bar{\theta}_{12}^{\prime}, 0\right) \\
& =R_{23}\left(\theta_{23}, 0\right) R_{13}\left(\bar{\theta}_{13}^{\prime}, \delta\right) R_{12}\left(\bar{\theta}_{12}^{\prime}, 0\right) \mathcal{Q},
\end{aligned}
$$

where we have defined

$$
\bar{\theta}_{13}^{\prime} \equiv \theta_{13}+\bar{\phi}^{\prime}
$$

This angle can be calculated directly without calculation $\bar{\phi}^{\prime}$ via

$$
\tan 2 \bar{\theta}_{13}^{\prime}=\frac{\left(\delta m_{31}^{2}-\delta m_{21}^{2} s_{12}^{2}\right) \sin 2 \theta_{13}}{\left(\delta m_{31}^{2}-\delta m_{21}^{2} s_{12}^{2}\right) \cos 2 \theta_{13}+a} .
$$

The phase matrix $\mathcal{Q}$ appearing rightmost in the above matrix product can be absorbed into the redefinition of the major phases and can be dropped. Thus, we arrive at our final approximation in which the vacuum mixing angles are replaced by their effective values in matter

$$
\begin{aligned}
\theta_{12} & \rightarrow \bar{\theta}_{12}^{\prime}=\theta_{12}+\bar{\varphi}, \\
\theta_{13} & \rightarrow \bar{\theta}_{13}^{\prime}=\theta_{13}+\bar{\phi}^{\prime}, \\
\theta_{23} & \rightarrow \theta_{23}, \\
\delta & \rightarrow \delta,
\end{aligned}
$$

and the eigenvalues of the effective Hamiltonian are given by

$$
\begin{aligned}
& \bar{\lambda}_{1} \approx \bar{\lambda}_{\mp}^{\prime \prime}, \\
& \bar{\lambda}_{2} \approx \bar{\lambda}_{+}^{\prime}, \\
& \bar{\lambda}_{3} \approx \bar{\lambda}_{ \pm}^{\prime \prime} .
\end{aligned}
$$

Note that of the mixing angles, only $\theta_{12}$ and $\theta_{13}$ are shifted. $\theta_{23}$ and $\delta$ stay at their vacuum values. 


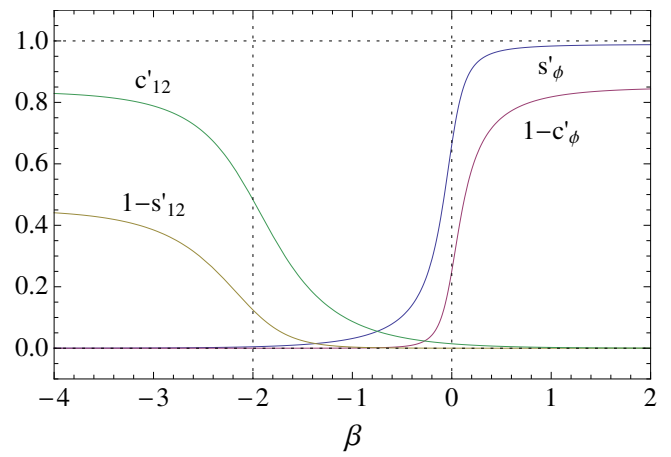

(a) Normal Hierarchy

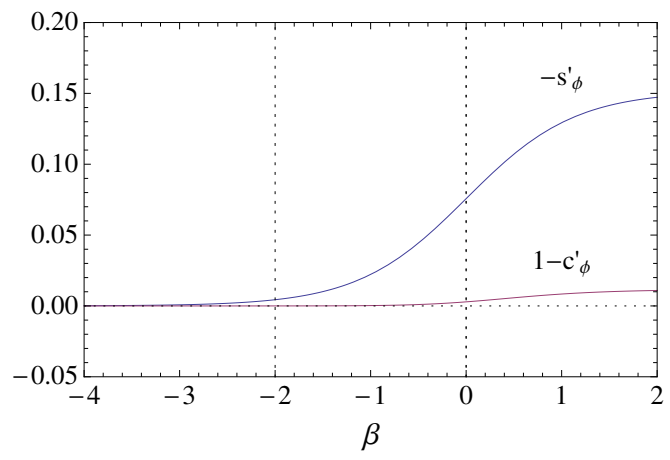

(b) Inverted Hierarchy

Figure 18. $\beta$-dependence of $c_{12}^{\prime}, 1-s_{12}^{\prime}, s_{\phi}^{\prime}$ and $1-c_{\phi}^{\prime}$ for (a) normal and (b) inverted hierarchies. The behaviors of $c_{12}^{\prime}$ and $1-s_{12}^{\prime}$ are common to both.

\section{Commutation of $R_{13}$ and $R_{23}$ through $R_{12}$}

In the derivation of our approximation formulae above, eqs. (B.31) and (B.59) played crucial roles in allowing the second rotation angle to be absorbed into $\theta_{13}$. In this appendix, we evaluate the validity of these approximations.

\section{C.1 Neutrino case}

The difference between the two sides of eq. (B.31) is given by

$$
\begin{aligned}
\delta R & \equiv R_{12}\left(\theta_{12}^{\prime}, 0\right) R_{23}\left(\phi^{\prime}, 0\right)-R_{13}\left(\phi^{\prime}, 0\right) R_{12}\left(\theta_{12}^{\prime}, 0\right) \\
& =\left[\begin{array}{ccc}
c_{12}^{\prime}\left(1-c_{\phi}^{\prime}\right) & 0 & -\left(1-s_{12}^{\prime}\right) s_{\phi}^{\prime} \\
0 & -c_{12}^{\prime}\left(1-c_{\phi}^{\prime}\right) & c_{12}^{\prime} s_{\phi}^{\prime} \\
c_{12}^{\prime} s_{\phi}^{\prime} & -\left(1-s_{12}^{\prime}\right) s_{\phi}^{\prime} & 0
\end{array}\right] .
\end{aligned}
$$

It is clear that $\delta R$ will vanish in the two limits $a \rightarrow 0$ where $s_{12}^{\prime} \rightarrow s_{12}, c_{12}^{\prime} \rightarrow c_{12}, s_{\phi}^{\prime} \rightarrow 0$, and $c_{\phi}^{\prime} \rightarrow 1$, and $a \rightarrow \infty$ where $s_{12}^{\prime} \rightarrow 1, c_{12}^{\prime} \rightarrow 0, s_{\phi}^{\prime} \rightarrow c_{13}\left(-s_{13}\right)$, and $c_{\phi}^{\prime} \rightarrow s_{13}\left(c_{13}\right)$ for normal(inverted) hierarchy. The question is whether $\delta R$ will stay negligible in between as $s_{12}^{\prime}$ runs from $s_{12}$ to $1, c_{12}^{\prime}$ from $c_{12}$ to $0, s_{\phi}^{\prime}$ from 0 to $c_{13}$ (normal) or $-s_{13}$ (inverted), and $c_{\phi}^{\prime}$ from 1 to $s_{13}$ (normal) or $c_{13}$ (inverted) as shown in figure 18. The dependence of the non-zero elements of $\delta R$ on $\beta=-\log _{\varepsilon}\left(a /\left|\delta m_{31}^{2}\right|\right)$ is shown in figure 19. The bumps at $a \sim \delta m_{21}^{2}$ for both hierarchies, and that at $a \sim \delta m_{31}^{2}$ for the normal hierarchy, happen due to the $\theta_{12}^{\prime}$ factor competing with the $\phi^{\prime}$ factor as one of them goes through a resonance while the other damps to zero. The heights of the bumps depend on the narrowness of the resonances.

For the case shown in figure 19, which was generated with the numbers in table 1 as input, all elements of $\delta R$ are $O\left(\varepsilon^{3}\right)$ or smaller for the entire range of $a$, with the maximum value of $\sim 0.01 \approx 2 \varepsilon^{3}$ occurring in $c_{12}^{\prime} s_{\phi}^{\prime}$ near $a \sim \delta m_{31}^{2}$ in the normal hierarchy case. Since the size of the third rotation angle we neglected in the Jacobi procedure was $O\left(\varepsilon^{2} s_{13}\right)$, eq. (B.31) is valid to the same order provided $s_{13}=O(\varepsilon)$. 


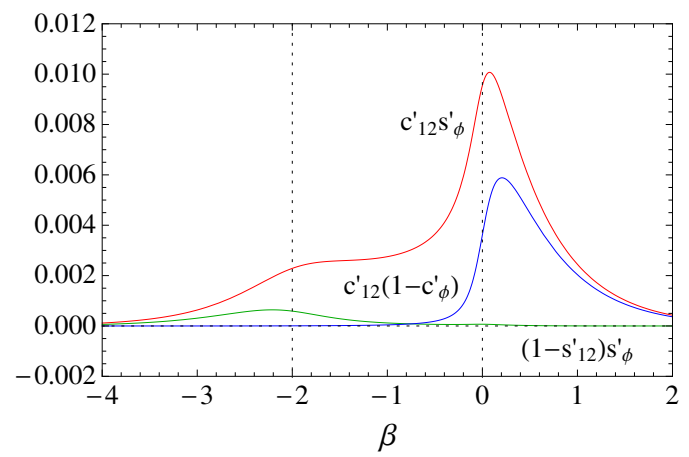

(a) Normal Hierarchy

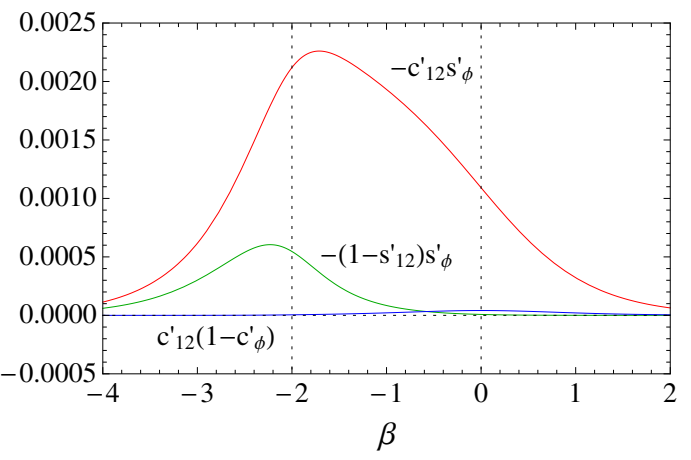

(b) Inverted Hierarchy

Figure 19. $\beta$-dependence of the non-zero elements of $\delta R$ for (a) normal and (b) inverted hierarchies.

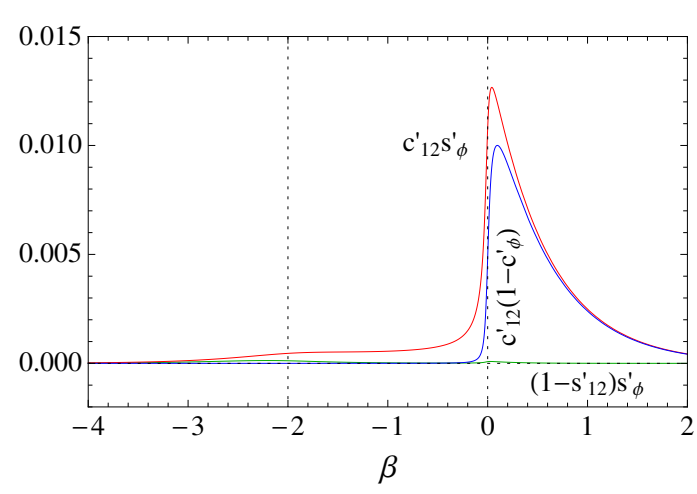

(a) $s_{13}=0.03$, Normal Hierarchy

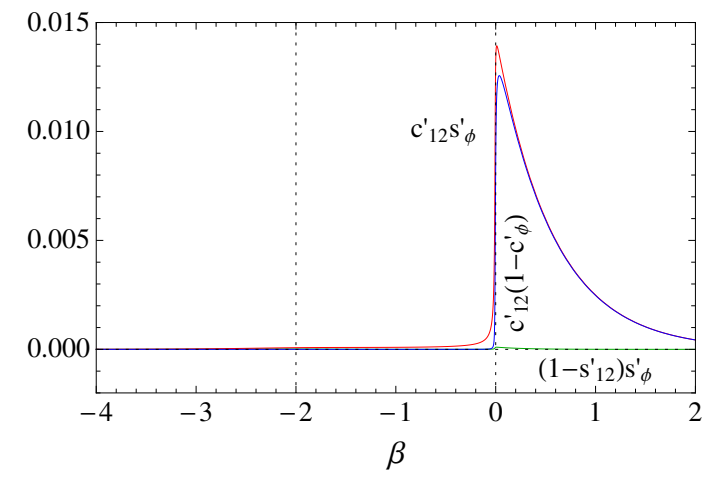

(b) $s_{13}=0.005$, Normal Hierarchy

Figure 20. $\beta$-dependence of the non-zero elements of $\delta R$ for different values of $s_{13}$ with normal hierarchy. (a) $s_{13}=0.03=O\left(\varepsilon^{2}\right)$, (b) $s_{13}=0.005=O\left(\varepsilon^{3}\right)$.

For smaller values of $s_{13}$, the resonance at $a \sim \delta m_{31}^{2}$ would have been narrower, and the peaks in $c_{12}^{\prime} s_{\phi}^{\prime}$ and $c_{12}^{\prime}\left(1-c_{\phi}^{\prime}\right)$ higher. This is illustrated in figure 20 . In the limit $s_{13} \rightarrow+0, s_{\phi}^{\prime}$ and $1-c_{\phi}^{\prime}$ will become step functions at $\beta \sim 0$, and the maximum height of the peak will be

$$
c_{12}^{\prime}\left(a \sim \delta m_{31}^{2}\right) \approx s_{12} c_{12} \varepsilon^{2}=0.46 \varepsilon^{2}=0.014,
$$

as can be discerned from eq. (B.17). This is the same as the asymptotic value of $a c_{12}^{\prime} / \delta m_{31}^{2}$ discussed earlier. While this value may not seem particularly large, only a factor of $3 / 2$ larger than the peak in figure 19(a), it is parametrically $O\left(\varepsilon^{2}\right)$. On the other hand, the third rotation angle neglected in the Jacobi procedure was $O\left(\varepsilon^{2} s_{13}\right)$. Thus, using eq. (B.31) would lead to dropping terms that are larger than the ones we keep when $s_{13}=O\left(\varepsilon^{2}\right)$ or smaller. Also, the sudden change in the accuracy of eq. (B.31) across $a \sim \delta m_{31}^{2}$, as can be seen in figure 20, will lead to kinks in the resulting oscillation probabilities. 


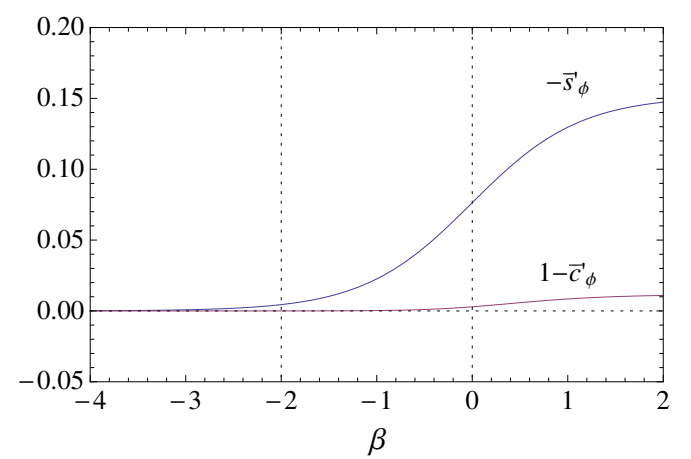

(a) Normal Hierarchy

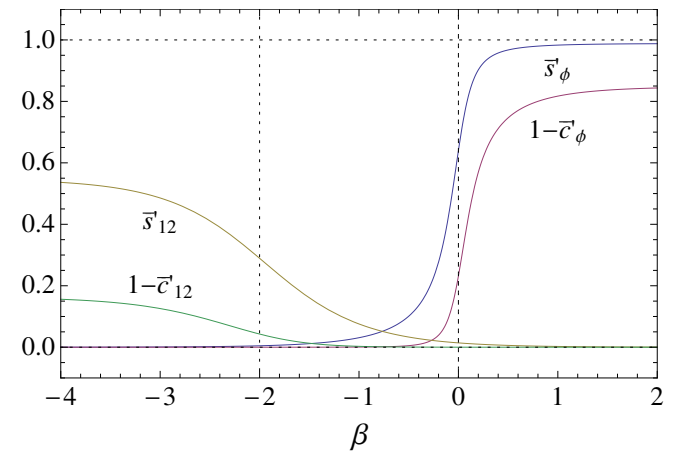

(b) Inverted Hierarchy

Figure 21. $\beta$-dependence of $\bar{s}_{12}^{\prime}, 1-\bar{c}_{12}^{\prime}, s_{\phi}^{\prime}$ and $1-c_{\phi}^{\prime}$ for (a) normal and (b) inverted hierarchies. The behaviors of $\bar{s}_{12}^{\prime}$ and $1-\bar{c}_{12}^{\prime}$ are common to both.

\section{C.2 Anti-neutrino case}

The difference between the two sides of eq. (B.59) is given by

$$
\begin{aligned}
\overline{\delta R} & \equiv R_{12}\left(\bar{\theta}_{12}^{\prime}, 0\right) R_{13}\left(\bar{\phi}^{\prime}, 0\right)-R_{13}\left(\bar{\phi}^{\prime}, 0\right) R_{12}\left(\bar{\theta}_{12}^{\prime}, 0\right) \\
& =\left[\begin{array}{ccc}
0 & \bar{s}_{12}^{\prime}\left(1-\bar{c}_{\phi}^{\prime}\right)-\left(1-\bar{c}_{12}^{\prime}\right) \bar{s}_{\phi}^{\prime} \\
\bar{s}_{12}^{\prime}\left(1-\bar{c}_{\phi}^{\prime}\right) & 0 & -\bar{s}_{12}^{\prime} \bar{s}_{\phi}^{\prime} \\
\left(1-\bar{c}_{12}^{\prime}\right) \bar{s}_{\phi}^{\prime} & \bar{s}_{12}^{\prime} \bar{s}_{\phi}^{\prime} & 0
\end{array}\right] .
\end{aligned}
$$

It is clear that $\overline{\delta R}$ will vanish in the two limits $a \rightarrow 0$ where $\bar{s}_{12}^{\prime} \rightarrow s_{12}, \bar{c}_{12}^{\prime} \rightarrow c_{12}, \bar{s}_{\phi}^{\prime} \rightarrow 0$, and $\bar{c}_{\phi}^{\prime} \rightarrow 1$, and $a \rightarrow \infty$ where $\bar{s}_{12}^{\prime} \rightarrow 0, \bar{c}_{12}^{\prime} \rightarrow 1, \bar{s}_{\phi}^{\prime} \rightarrow-s_{13}\left(c_{13}\right)$, and $\bar{c}_{\phi}^{\prime} \rightarrow c_{13}\left(s_{13}\right)$ for normal(inverted) hierarchy. The question is whether $\delta R$ will stay negligible in between as $\bar{s}_{12}^{\prime}$ runs from $s_{12}$ to $0, \bar{c}_{12}^{\prime}$ from $c_{12}$ to $1, \bar{s}_{\phi}^{\prime}$ from 0 to $-s_{13}$ (normal) or $c_{13}$ (inverted), and $\bar{c}_{\phi}^{\prime}$ from 1 to $c_{13}$ (normal) or $s_{13}$ (inverted) as shown in figure 21 .

The dependence of the non-zero elements of $\overline{\delta R}$ on $\beta=-\log _{\varepsilon}\left(a /\left|\delta m_{31}^{2}\right|\right)$ is shown in figure 22 , which was generated with the numbers in table 1 as input. We can see that all elements of $\overline{\delta R}$ are $O\left(\varepsilon^{3}\right)$ or smaller for the entire range of $a$, with the maximum value of $\sim 0.01 \approx 2 \varepsilon^{3}$ occuring in $\bar{s}_{12}^{\prime} \bar{s}_{\phi}^{\prime}$ near $a \sim \delta m_{31}^{2}$ in the inverted hierarchy case. Since the size of the third rotation angle we neglected in the Jacobi procedure was $O\left(\varepsilon^{2} s_{13}\right)$, eq. (B.59) is valid to the same order provided $s_{13}=O(\varepsilon)$.

For smaller values of $s_{13}$, the resonance at $a \sim \delta m_{31}^{2}$ would have been narrower, and the peaks in $\bar{s}_{12}^{\prime} \bar{s}_{\phi}^{\prime}$ and $\bar{s}_{12}^{\prime}\left(1-\bar{c}_{\phi}^{\prime}\right)$ higher. This is illustrated in figure 23 . In the limit $s_{13} \rightarrow+0, \bar{s}_{\phi}^{\prime}$ and $1-\bar{c}_{\phi}^{\prime}$ will become step functions at $\beta \sim 0$, and the maximum height of the peak will be the same as eq. (C.2), and the asymptotic value of $a \bar{s}_{12}^{\prime} /\left|\delta m_{31}^{2}\right|$, as can be discerned from eq. (B.46). This is parametrically $O\left(\varepsilon^{2}\right)$, while the third rotation angle neglected in the Jacobi procedure was $O\left(\varepsilon^{2} s_{13}\right)$. Thus, using eq. (B.59) would lead to dropping terms that are larger than the ones we keep when $s_{13}=O\left(\varepsilon^{2}\right)$ or smaller. Also, the sudden change in the accuracy of eq. (B.59) across $a \sim \delta m_{31}^{2}$, as can be seen in figure 23, will lead to kinks in the resulting oscillation probabilities. 


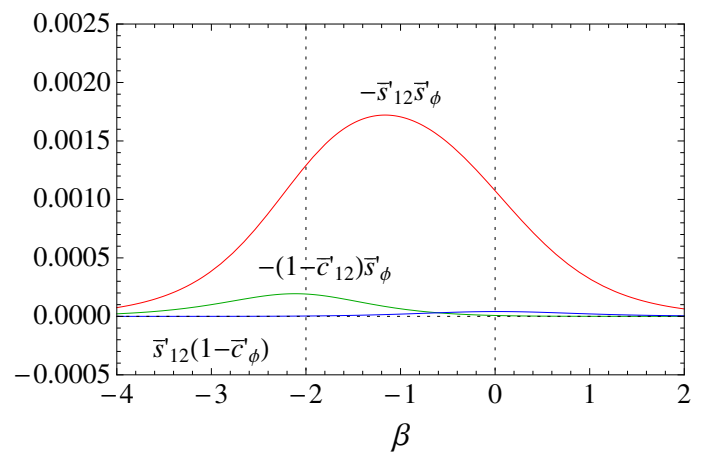

(a) Normal Hierarchy

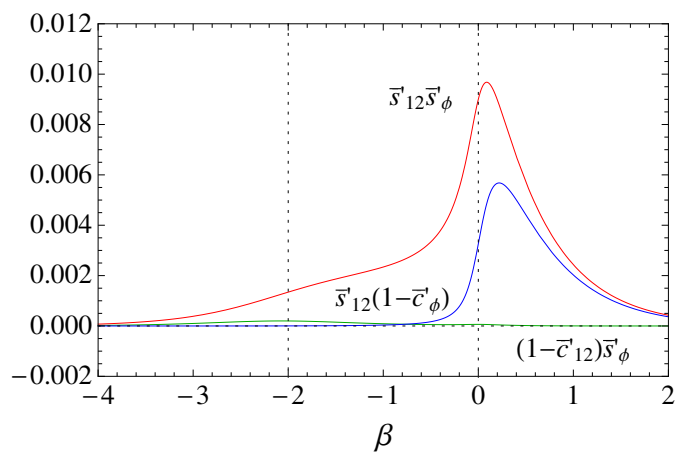

(b) Inverted Hierarchy

Figure 22. $\beta$-dependence of the non-zero elements of $\overline{\delta R}$ for (a) normal and (b) inverted hierarchies.

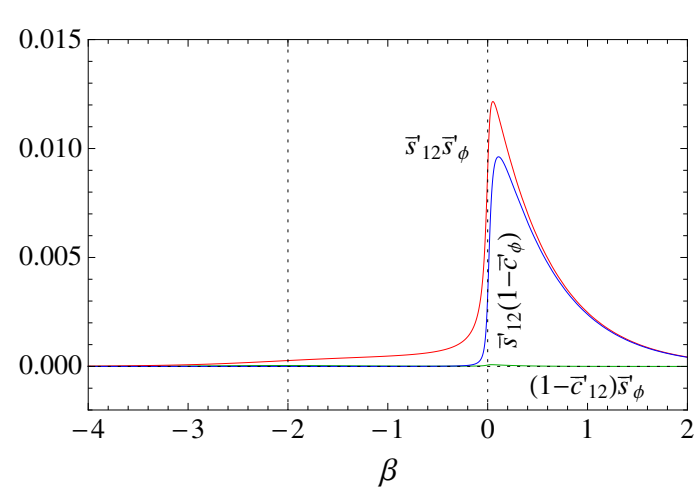

(a) $s_{13}=0.03$, Inverted Hierarchy

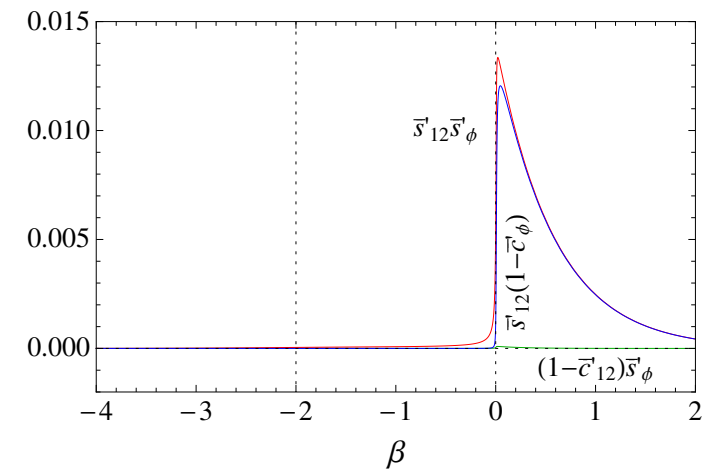

(b) $s_{13}=0.005$, Inverted Hierarchy

Figure 23. $\beta$-dependence of the non-zero elements of $\overline{\delta R}$ for different values of $s_{13}$ with inverted hierarchy. (a) $s_{13}=0.03=O\left(\varepsilon^{2}\right)$, (b) $s_{13}=0.005=O\left(\varepsilon^{3}\right)$.

Open Access. This article is distributed under the terms of the Creative Commons Attribution License (CC-BY 4.0), which permits any use, distribution and reproduction in any medium, provided the original author(s) and source are credited.

\section{References}

[1] H. Minakata and H. Nunokawa, CP violation versus matter effect in long baseline neutrino oscillation experiments, Phys. Rev. D 57 (1998) 4403 [hep-ph/9705208] [INSPIRE].

[2] M.C. Banuls, G. Barenboim and J. Bernabeu, Medium effects for terrestrial and atmospheric neutrino oscillations, Phys. Lett. B 513 (2001) 391 [hep-ph/0102184] [INSPIRE].

[3] P. Huber, M. Lindner and W. Winter, Superbeams versus neutrino factories, Nucl. Phys. B 645 (2002) 3 [hep-ph/0204352] [INSPIRE].

[4] R. Gandhi, P. Ghoshal, S. Goswami, P. Mehta and S.U. Sankar, Earth matter effects at very long baselines and the neutrino mass hierarchy, Phys. Rev. D 73 (2006) 053001 [hep-ph/0411252] [INSPIRE]. 
[5] P. Huber, M. Maltoni and T. Schwetz, Resolving parameter degeneracies in long-baseline experiments by atmospheric neutrino data, Phys. Rev. D 71 (2005) 053006 [hep-ph/0501037] [INSPIRE].

[6] E.K. Akhmedov, M. Maltoni and A.Y. Smirnov, 1-3 leptonic mixing and the neutrino oscillograms of the Earth, JHEP 05 (2007) 077 [hep-ph/0612285] [INSPIRE].

[7] S.K. Agarwalla, T. Li, O. Mena and S. Palomares-Ruiz, Exploring the Earth matter effect with atmospheric neutrinos in ice, arXiv:1212.2238 [INSPIRE].

[8] M. Blennow and A.Y. Smirnov, Neutrino propagation in matter, Adv. High Energy Phys. 2013 (2013) 972485 [arXiv:1306.2903] [INSPIRE].

[9] L. Wolfenstein, Neutrino Oscillations in Matter, Phys. Rev. D 17 (1978) 2369 [InSPIRE].

[10] S.P. Mikheev and A.Y. Smirnov, Resonance Amplification of Oscillations in Matter and Spectroscopy of Solar Neutrinos, Sov. J. Nucl. Phys. 42 (1985) 913 [InSPIRE].

[11] S.P. Mikheev and A.Y. Smirnov, Resonant amplification of neutrino oscillations in matter and solar neutrino spectroscopy, Nuovo Cim. C 9 (1986) 17 [INSPIRE].

[12] V.D. Barger, K. Whisnant, S. Pakvasa and R.J.N. Phillips, Matter Effects on Three-Neutrino Oscillations, Phys. Rev. D 22 (1980) 2718 [InSPIRE].

[13] S. Toshev, Resonant Amplification of Three Neutrino Oscillations in Matter, Phys. Lett. B 185 (1987) 177 [Erratum ibid. B 192 (1987) 478] [INSPIRE].

[14] S.T. Petcov and S. Toshev, Three Neutrino Oscillations in Matter: Analytical Results in the Adiabatic Approximation, Phys. Lett. B 187 (1987) 120 [INSPIRE].

[15] C.W. Kim and W.K. Sze, Adiabatic Resonant Oscillations of Solar Neutrinos in Three Generations, Phys. Rev. D 35 (1987) 1404 [INSPIRE].

[16] H.W. Zaglauer and K.H. Schwarzer, The Mixing Angles in Matter for Three Generations of Neutrinos and the MSW Mechanism, Z. Phys. C 40 (1988) 273 [INSPIRE].

[17] P.I. Krastev and S.T. Petcov, Resonance Amplification and $t$ Violation Effects in Three Neutrino Oscillations in the Earth, Phys. Lett. B 205 (1988) 84 [INSPIRE].

[18] S. Toshev, On T violation in matter neutrino oscillations, Mod. Phys. Lett. A 6 (1991) 455 [INSPIRE].

[19] K. Dick, M. Freund, M. Lindner and A. Romanino, CP violation in neutrino oscillations, Nucl. Phys. B 562 (1999) 29 [hep-ph/9903308] [InSPIRE].

[20] T. Ohlsson and H. Snellman, Three flavor neutrino oscillations in matter, J. Math. Phys. 41 (2000) 2768 [Erratum ibid. 42 (2001) 2345] [hep-ph/9910546] [INSPIRE].

[21] T. Ohlsson and H. Snellman, Neutrino oscillations with three flavors in matter: Applications to neutrinos traversing the Earth, Phys. Lett. B 474 (2000) 153 [hep-ph/9912295] [INSPIRE].

[22] M. Freund, Analytic approximations for three neutrino oscillation parameters and probabilities in matter, Phys. Rev. D 64 (2001) 053003 [hep-ph/0103300] [INSPIRE].

[23] K. Kimura, A. Takamura and H. Yokomakura, Exact formulas and simple CP dependence of neutrino oscillation probabilities in matter with constant density, Phys. Rev. D 66 (2002) 073005 [hep-ph/0205295] [INSPIRE].

[24] G. Cardano, Ars Magna, originally published in 1545, translated from Latin by T. Richard Witmer, The Rules of Algebra, Dover (2007). 
[25] T.-K. Kuo and J.T. Pantaleone, The Solar Neutrino Problem and Three Neutrino Oscillations, Phys. Rev. Lett. 57 (1986) 1805 [InSPIRE].

[26] J. Arafune, M. Koike and J. Sato, CP violation and matter effect in long baseline neutrino oscillation experiments, Phys. Rev. D 56 (1997) 3093 [Erratum ibid. D 60 (1999) 119905] [hep-ph/9703351] [INSPIRE].

[27] A. Cervera et al., Golden measurements at a neutrino factory, Nucl. Phys. B 579 (2000) 17 [Erratum ibid. B 593 (2001) 731] [hep-ph/0002108] [INSPIRE].

[28] O.L.G. Peres and A.Y. Smirnov, Atmospheric neutrinos: LMA oscillations, U(e3) induced interference and CP-violation, Nucl. Phys. B 680 (2004) 479 [hep-ph/0309312] [InSPIRE].

[29] E.K. Akhmedov, R. Johansson, M. Lindner, T. Ohlsson and T. Schwetz, Series expansions for three flavor neutrino oscillation probabilities in matter, JHEP 04 (2004) 078 [hep-ph/0402175] [INSPIRE].

[30] E.K. Akhmedov, M.A. Tortola and J.W.F. Valle, A Simple analytic three flavor description of the day night effect in the solar neutrino flux, JHEP 05 (2004) 057 [hep-ph/0404083] [INSPIRE].

[31] E.K. Akhmedov and V. Niro, An Accurate analytic description of neutrino oscillations in matter, JHEP 12 (2008) 106 [arXiv:0810.2679] [INSPIRE].

[32] K. Asano and H. Minakata, Large-Theta(13) Perturbation Theory of Neutrino Oscillation for Long-Baseline Experiments, JHEP 06 (2011) 022 [arXiv:1103.4387] [INSPIRE].

[33] DAYA-BAY collaboration, F.P. An et al., Observation of electron-antineutrino disappearance at Daya Bay, Phys. Rev. Lett. 108 (2012) 171803 [arXiv:1203.1669] [INSPIRE].

[34] DAYA BAY collaboration, F.P. An et al., Improved Measurement of Electron Antineutrino Disappearance at Daya Bay, Chin. Phys. C 37 (2013) 011001 [arXiv:1210.6327] [inSPIRE].

[35] RENO collaboration, J.K. Ahn et al., Observation of Reactor Electron Antineutrino Disappearance in the RENO Experiment, Phys. Rev. Lett. 108 (2012) 191802 [arXiv: 1204.0626] [INSPIRE].

[36] T2K collaboration, K. Abe et al., Indication of Electron Neutrino Appearance from an Accelerator-produced Off-axis Muon Neutrino Beam, Phys. Rev. Lett. 107 (2011) 041801 [arXiv:1106.2822] [INSPIRE].

[37] MINOS collaboration, P. Adamson et al., Improved search for muon-neutrino to electron-neutrino oscillations in MINOS, Phys. Rev. Lett. 107 (2011) 181802 [arXiv:1108.0015] [INSPIRE].

[38] MINOS collaboration, P. Adamson et al., Electron neutrino and antineutrino appearance in the full MINOS data sample, Phys. Rev. Lett. 110 (2013) 171801 [arXiv:1301.4581] [INSPIRE].

[39] Double CHOOZ collaboration, Y. Abe et al., Indication for the disappearance of reactor electron antineutrinos in the Double CHOOZ experiment, Phys. Rev. Lett. 108 (2012) 131801 [arXiv: 1112.6353] [INSPIRE].

[40] Double CHOOZ collaboration, Y. Abe et al., Reactor electron antineutrino disappearance in the Double CHOOZ experiment, Phys. Rev. D 86 (2012) 052008 [arXiv:1207.6632] [INSPIRE]. 
[41] M.C. Gonzalez-Garcia, M. Maltoni, J. Salvado and T. Schwetz, Global fit to three neutrino mixing: critical look at present precision, JHEP 12 (2012) 123 [arXiv:1209.3023] [INSPIRE].

[42] M. Honda, Y. Kao, N. Okamura and T. Takeuchi, A Simple parameterization of matter effects on neutrino oscillations, hep-ph/0602115 [INSPIRE].

[43] M. Honda, N. Okamura and T. Takeuchi, Matter Effect on Neutrino Oscillations from the violation of Universality in Neutrino Neutral Current Interactions, hep-ph/0603268 [INSPIRE].

[44] C.G.J. Jacobi, Über ein leichtes Verfahren, die in der Theorie der Säkularstörangen vorkommenden Gleichungen numerisch Aufzuloösen, Crelle's Journal 30 (1846) 51.

[45] A.M. Dziewonski and D.L. Anderson, Preliminary reference earth model, Physics of the Earth and Planetary Interiors 25 (1981) 297.

[46] W. Liao, Precise Formulation of Neutrino Oscillation in the Earth, Phys. Rev. D 77 (2008) 053002 [arXiv: 0710 . 1492] [INSPIRE].

[47] S.K. Agarwalla and P. Hernández, Probing the Neutrino Mass Hierarchy with Super-Kamiokande, JHEP 10 (2012) 086 [arXiv:1204.4217] [INSPIRE].

[48] A. Stahl et al., Expression of Interest for a very long baseline neutrino oscillation experiment (LBNO), CERN-SPSC-2012-021.

[49] P. Huber and W. Winter, Neutrino factories and the 'magic' baseline, Phys. Rev. D 68 (2003) 037301 [hep-ph/0301257] [INSPIRE].

[50] A.Y. Smirnov, Neutrino oscillations: What is 'magic' about the magic baseline?, hep-ph/0610198 [INSPIRE].

[51] M. Honda, Y. Kao, N. Okamura, A. Pronin and T. Takeuchi, Constraints on New Physics from Matter Effects on Neutrino Oscillation, hep-ph/0610281 [INSPIRE].

[52] M. Honda, Y. Kao, N. Okamura, A. Pronin and T. Takeuchi, The Effect of Topcolor Assisted Technicolor and other models, on Neutrino Oscillation, arXiv:0704.0369 [INSPIRE].

[53] M. Honda, Y. Kao, N. Okamura, A. Pronin and T. Takeuchi, Constraints on New Physics from Long Baseline Neutrino Oscillation Experiments, arXiv:0707.4545 [INSPIRE].

[54] B. Pontecorvo, Inverse beta processes and nonconservation of lepton charge, Sov. Phys. JETP 7 (1958) 172 [INSPIRE].

[55] Z. Maki, M. Nakagawa and S. Sakata, Remarks on the unified model of elementary particles, Prog. Theor. Phys. 28 (1962) 870 [INSPIRE].

[56] B. Pontecorvo, Neutrino Experiments and the Problem of Conservation of Leptonic Charge, Sov. Phys. JETP 26 (1968) 984 [inSPIRE].

[57] K. Hagiwara and N. Okamura, Quark and lepton flavor mixings in the SU(5) grand unification theory, Nucl. Phys. B 548 (1999) 60 [hep-ph/9811495] [INSPIRE].

[58] K. Nakamura and S.T. Petkov, Neutrino mass, mixing, and oscillations, Phys. Rev. D 86 (2012) 177.

[59] C. Jarlskog, Commutator of the Quark Mass Matrices in the Standard Electroweak Model and a Measure of Maximal CP-violation, Phys. Rev. Lett. 55 (1985) 1039 [INSPIRE].

[60] Particle Data Group collaboration, J. Beringer et al., Review of Particle Physics (RPP), Phys. Rev. D 86 (2012) 010001 [inSPIRE]. 